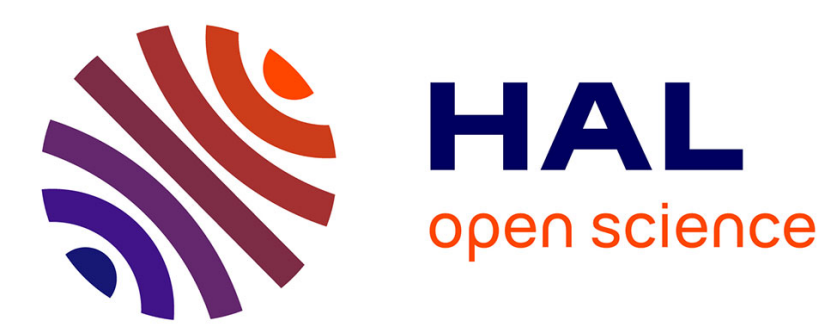

\title{
A unified single-field Volume-of-Fluid-based formulation for multi-component interfacial transfer with local volume changes
}

Julien Maes, Cyprien Soulaine

\section{- To cite this version:}

Julien Maes, Cyprien Soulaine. A unified single-field Volume-of-Fluid-based formulation for multicomponent interfacial transfer with local volume changes. Journal of Computational Physics, 2020, 402, pp.109024. 10.1016/j.jcp.2019.109024 . insu-02426952

\section{HAL Id: insu-02426952}

\section{https://hal-insu.archives-ouvertes.fr/insu-02426952}

Submitted on 24 Nov 2020

HAL is a multi-disciplinary open access archive for the deposit and dissemination of scientific research documents, whether they are published or not. The documents may come from teaching and research institutions in France or abroad, or from public or private research centers.
L'archive ouverte pluridisciplinaire HAL, est destinée au dépôt et à la diffusion de documents scientifiques de niveau recherche, publiés ou non, émanant des établissements d'enseignement et de recherche français ou étrangers, des laboratoires publics ou privés. 


\title{
A unified single-field Volume-of-Fluid-based formulation for multi-component interfacial transfer with local volume changes
}

\author{
Julien Maes ${ }^{\mathrm{a}}$, Cyprien Soulaine ${ }^{\mathrm{b}}$ \\ ${ }^{a}$ Institute of GeoEnergy Engineering, Heriot-Watt University, Edinburgh, U.K. \\ ${ }^{b}$ French Geological Survey, BRGM, Orléans, France
}

\section{Abstract}

7 This paper presents a novel unified single-field formulation for Volume-Of-Fluid simulation of interfacial mass transfer with local volume changes. By comparison with the previous models referred as Continuous Species Transfer in the literature, our improved model uses a single-field formulation of the local mass transfer across the interface, enabling us to take into account local volume changes induced by non-dilute species transfer. The numerical model, implemented in our in-house OpenFOAM-based simulator, is validated by comparison with analytical solutions in 1D and 2D, and a semi-analytical solution in 3D. The implemented approach is first applied to investigate competing mass transfer in an infinite cylinder. We then simulate the shrinking of a single-component rising bubble at low Schmidt number. The numerical model is shown to be well adapted to investigate local Sherwood numbers and existing correlation for mass transfer at fluid interfaces.

Keywords: Interface, Mass transfer, Multicomponent fluid, Local volume change, Volume-Of-Fluid, OpenFOAM

\section{1. Introduction}

Interface species transfers are present in a wide range of applications such as acid gas treatment, bubble column reactors and geological storage of $\mathrm{CO}_{2}$ in aquifers. These processes include viscous and capillary effects, chemical reactions and the coupling between mass transfer and fluid dynamics. For such complex systems, analytical solution are only restricted to very simple geometries and flow conditions (Danckwerts, 1970; Hadamard, 1911; Coutelieris et al., 2006). In addition, experimental studies are often time-consuming, hard to control, and quantities of interest may be difficult to measure during the course of the experiment. Numerical simulation is therefore a powerful tool to investigate these processes and achieve optimal design and process control.

Numerical simulation of interfacial mass transfer can be done using Direct Numerical Simulation (DNS) of the Navier-Stokes Equation (NSE), for which mass and momentum conservation are directly solved without model simplification. DNS of two-phase flow can be performed using the Volume-Of-Fluid (VOF) method, for which the interface between the two fluids is captured using an indicator function, which is a phase volume fraction (Hirt and Nichols, 1981). Although other methods such as level-set (Sussman et al., 1994; Chai et al., 2017; Gibou et al., 2018; Luo et al., 2019) can provide a more accurate description of the sharp interface, the VOF method is attractive due to its flexibility, robustness in terms of mass conservation, and adaptability to more complex physic.

VOF methods can be geometric or algebraic, depending on how the computation of the interface curvature and the advection of the indicator function are performed. Geometric methods explicitly reconstruct the interface, and the advection of the indicator function is performed based on a geometric representation of the faces flux (Gerlach et al., 2006; Weymouth and Yue, 2010; Owkes and Desjardins, 2014). Geometric methods do not create numerical diffusion and can achieve better precision with accurate interface reconstruction (Popinet, 2009), but their application to unstructured mesh is highly demanding and scarcely used (Maric et al., 2013). Alternatively, algebraic methods perform the advection of the indicator function by numerically solving a transport equation 
(Hirt and Nichols, 1981). Algebraic methods can be easily applied to unstructured grids as no explicit interface reconstruction is needed. The main challenge is to preserve a sharp interface while maintaining the boundedness of the phase volume fraction. High resolution differencing schemes and models with compression of the interface are generally introduced to reduce this problem (Zalesak, 1979; Ubbink and Issa, 1999).

To simulate species transfer in two-phase systems within the VOF method, two conceptually different approaches have been developed, namely the two-field and single-field approaches. The main criterion to decide which approach to use is to ensure consistency of the advection operator for phase fraction and species concentration, otherwise artificial mass transfer arises (Deising et al., 2016). In the two-field approach, the species concentration in each phase is solved separately, using two concentration fields - one per phase - which are set to zero when continued in the other phase (Bothe and Fleckenstein, 2013). Consistent advection of phase fraction and species concentration can only be achieved if the interface is geometrically advected. Therefore, the two-field approach is consistent in principles with any geometric VOF method. In the single-field approach, a mixture quantity, obtain by volume averaging of species concentration, is transported by solving an algebraic equation (Haroun et al., 2010; Deising et al., 2016). Therefore, the single-field approach is consistent in principles with interface advection for any algebraic VOF method, and has been applied with high resolution differencing schemes (Haroun et al., 2010; Deising et al., 2016, 2018) and models with compression of the interface (Yang et al., 2017; Graveleau et al., 2017; Maes and Soulaine, 2018). Hence, if a geometric VOF method is employed, the two-field approach should be used. On the other side, if an algebraic VOF method is employed, then the single-field approach should be used.

Both two-field and single-field approaches exhibit method-specific advantages and disadvantages. Within the two-field approach, a sub-grid model based on the reconstructed interface can be used to reduce the resolution required around the interface (Bothe and Fleckenstein, 2013). Within the single-field approach, the inclusion of sub-grid scale models is complex and requires further research. Another benefit of the two-field approach is that the concentration gradients are readily available, which allows for calculating local mass transfer across the interface in a straightforward manner, and enabled Fleckenstein and Bothe (2015) to extend the model to include local volume changes for non-dilute mass transfer. However, since the two-field approach is only consistent with geometric VOF method, applying the method to complex unstructured grids is challenging. On the other side, applying the single-field approach on unstructured grids is straightforward, since it is consistent in principles with any algebraic VOF method (Marschall et al., 2012; Deising et al., 2016). This is essential for some application such as pore-scale simulation of $\mathrm{CO}_{2}$ storage in the subsurface (Graveleau et al., 2017) or pore-scale simulation of enhanced oil recovery (Maes and Geiger, 2018). Another advantage of the single-field approach is that it is fully consistent with the micro-continuum formulation for modelling multiscale transport in porous media (Soulaine et al., 2018, 2019)

However, no model exists to include local volume changes for non-dilute mass transfer within the single-field approach. Contrary to the two-field approach, the information about the one-sided interface concentrations and one-sided concentration gradients is inherently lost and need to be recovered with numerical modelling. Soh et al. (2017a,b) applied the two-field approach to an algebraic VOF model by reconstructing the phase concentration gradient at the interface. Although this formulation ensures continuity of the chemical potential, it does not ensure continuity of the species mass flux (convective and diffusive) across the interface, and artificial mass transfer arises. Up to now, there is no consistent model to compute the local mass transfer. The objective of the present work is to correct this problem in order to simulate interface transfer during two-phase flow with local volume changes using the single-field formulation. The main contribution is the derivation of a single-field numerical model of the interface transfer and local Sherwood number (Section 2.4) and its application to gas dissolution in liquid solvent, something that could only be done with the two-field approach before.

The paper is organised as follows. The governing equations and numerical models including the computation of the local mass transfer are presented in Section 2. In Section 3, the model is validated by comparison with 
analytical solutions in 1D and 2D and a semi-analytical solution in 3D. Finally, the model is applied to simulate competing mass transfer in an infinite cylinder and mass transfer during rising of a single-component gas bubble

at low Schmidt number.

\section{Mathematical model}

In this section, we introduce the model used to simulate two-phase flow with local volume change. First, we present the governing equations in the physical continuous domain. Then, we derive the grid-based VOF equations used to simulate the movement of a fluid/fluid interface along with multicomponent mass transfer on a discrete Eulerian mesh. Finally, we discuss the numerical implementation and the post-processing procedure.

\subsection{Governing equations in the continuous physical space}

This section presents the basic continuum hydrodynamic laws that govern multiphase flow with interface transfer. The domain is decomposed into two disjoint subsets, namely the disperse (gas) phase $\Omega_{d}$ and the continuous (liquid) phase $\Omega_{c}$, separated by the interface $\Sigma$ (see Figure 1a). Each phase is assumed to be Newtonian and in-

a) Continuous physical space

b) Grid-based domain
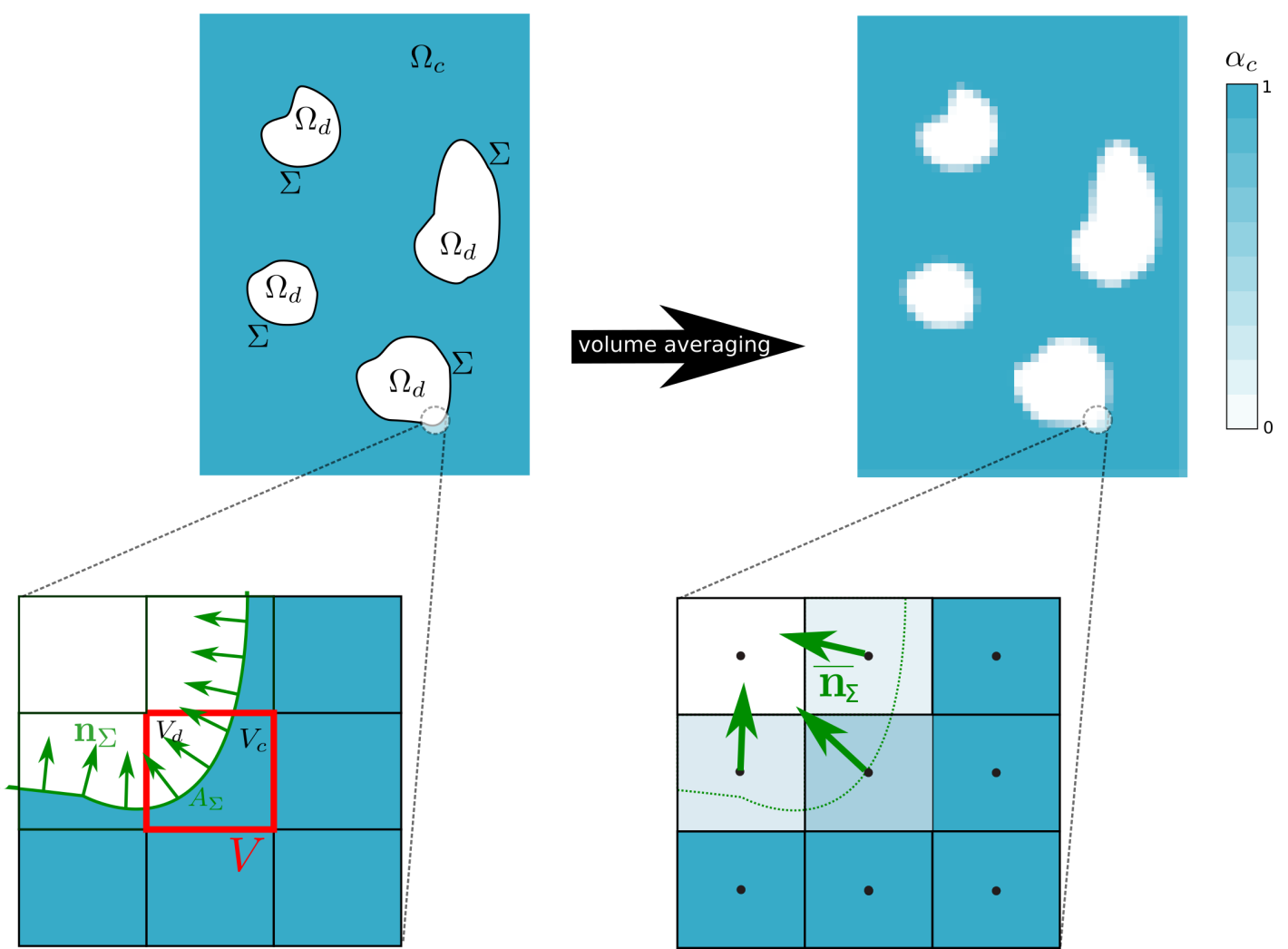

Figure 1: Distribution of the fluid phases in (a) the continuous physical domain, (b) the discrete Eulerian grid.

compressible, and fluid properties are assumed to be constant in each phase (in particular independent of the phase composition). Therefore, mass conservation in each phase writes

$$
\nabla \cdot \mathbf{u}_{i}=0 \quad \text { in } \Omega_{i}, i=d, c,
$$

where $\mathbf{u}_{i}$ is the velocity of phase $i$. Mass conservation at the fluid/fluid interface writes

$$
\llbracket \rho_{i}\left(\mathbf{u}_{i}-\mathbf{w}\right) \cdot \mathbf{n}_{\Sigma} \rrbracket=0 \quad \text { at } \Sigma,
$$


where $\rho_{i}$ is the density of phase $i, \mathbf{w}$ is the velocity of the interface, $\mathbf{n}_{\Sigma}$ is the normal vector to the fluid/fluid interface from the continuous to the disperse phase, and the double brackets describe a jump at the interface. In addition, the momentum conservation under the classical NSE formulation writes

$$
\rho_{i}\left(\frac{\partial \mathbf{u}_{i}}{\partial t}+\mathbf{u}_{i} \cdot \nabla \mathbf{u}_{i}\right)=-\nabla p_{i}+\nabla \cdot \mathrm{S}_{i}+\rho_{i} \mathbf{g} \quad \text { in } \Omega_{i}, i=d, c
$$

where $\mathbf{g}$ is the gravity vector, $\mathrm{S}_{i}=\mu_{i}\left(\nabla \mathbf{u}_{i}+\nabla \mathbf{u}_{i}^{T}\right)$ is the viscous stress tensor, and $p_{i}$ and $\mu_{i}$ are the pressure and viscosity of phase $i$, respectively. Momentum conservation at the fluid/fluid interface writes

$$
\llbracket p_{i} \mathrm{I}-\mathrm{S}_{i} \rrbracket \cdot \mathbf{n}_{\Sigma}=\sigma \kappa \mathbf{n}_{\Sigma} \quad \text { at } \Sigma,
$$

where $\mathrm{I}$ is the unity tensor, $\sigma$ is the interfacial tension and $\kappa=-\nabla \cdot \mathbf{n}_{\Sigma}$ is the interface curvature.

Both phases are assumed to be composed of a mixture of $n$ components. We assume that component $n$ is the solvent of the continuous phase, and that all other species present in the continuous phase are dilute. Furthermore, we assume that component $n$ is not soluble in the disperse phase. Therefore, the concentration $c_{j, i}$ of species $j$ in phase $i$ satisfies

$$
\sum_{1 \leq j<n} c_{j, d}=\rho_{d}, \quad \sum_{1 \leq j \leq n} c_{j, c}=\rho_{c} .
$$

In the absence of homogeneous chemical reactions, conservation of mass of species $j$ in phase $i$ is described by the standard advection-diffusion equation

$$
\frac{\partial c_{j, i}}{\partial t}+\nabla \cdot\left(c_{j, i} \mathbf{u}_{i}+\mathbf{J}_{j, i}\right)=0 \quad \text { in } \Omega_{i}
$$

where $\mathbf{J}_{j, i}$ is the mass flux of component $j$ in phase $i$ by diffusion. For all dilute components in the system, the diffusive mass flux can be modelled using Fick's law (Taylor and Krishna, 1993). This applies to every component in the continuous phase but component $n$. The diffusive flux of $n$ in phase $c$ can be obtained using

$$
\mathbf{J}_{n, c}=-\sum_{1 \leq j<n} \mathbf{J}_{j, c} .
$$

However, we do not need to solve the mass conservation of component $n$ since closure is obtained using the mass conservation of each phase (Eq. 1). Finally, we assume that the diffusive flux of species in the disperse phase can also be modelled using Fick's law. This is true for the two cases simulated here, namely a single-component and a binary disperse mixture. Therefore, for all components simulated in the system, the mass flux by diffusion can be modelled using

$$
\mathbf{J}_{j, i}=-D_{j, i} \nabla c_{j, i}
$$

where $D_{j, i}$ is the molecular diffusion coefficient of component $j$ in phase $i$. At the interface, mass conservation imposes continuity of mass fluxes on each side

$$
\llbracket\left(c_{j, i}\left(\mathbf{u}_{i}-\mathbf{w}\right)+\mathbf{J}_{j, i}\right) \cdot \mathbf{n}_{\Sigma} \rrbracket=0 \quad \text { at } \Sigma
$$

Finally, thermodynamics equilibrium imposes equality of chemical potential at the interface. This is model here using Henry's law

$$
c_{j, c}=H_{j} c_{j, d} \text { on } \Sigma,
$$

where $H_{j}$ is the Henry's coefficient for component $j$, assumed constant here for simplicity. 


\subsection{Algebraic VOF Method: from continuous to discrete domain}

The mathematical model introduced in the former section is defined on a continuous physical domain. The computational procedure to solve this system of equations, however, relies on the Finite Volume Method (FVM) (Patankar, 1980), i.e. a discretization of the domain into an ensemble of subset volumes. In FVM, the partial differential equations representing conservation laws (Eqs. (1), (3) and (6)) are transformed into discrete algebraic equations by integrating them over each discrete volume, $V$. This operation is carried out using the averaging operator,

$$
\overline{\beta_{i}}=\frac{1}{V} \int_{V_{i}} \beta_{i} d V,
$$

where $\beta_{i}$ is a function defined in $\Omega_{i}(i=c, d)$ and $V_{i}=V \cap \Omega_{i}$. The application of the volume average operator to the indicator function $\mathbb{1}_{i}$ (which is equal to 1 in $\Omega_{i}$ and 0 elsewhere) defines the volume fraction, $\alpha_{i}$, of phase $i$ in the finite volume $V$,

$$
\alpha_{i}=\frac{1}{V} \int_{V_{i}} \mathbb{1}_{i} d V .
$$

$\alpha_{i}$ is used as the phase indicator function in the FVM grid. In cells that contain the disperse phase only, $\alpha_{d}=1$. In cells that contain the continuous phase only, $\alpha_{d}=0$. In cells crossed by the fluid-fluid interface $\Sigma, 0<\alpha_{d}<1$ (see Figure 1b). Tracking the evolution of $\alpha_{i}$ is the cornerstone of the Volume-of-Fluid technique used in this paper. In VOF, this is achieved by solving conservation laws for the single-field (global variable) quantity, $\beta$, defined as

$$
\beta=\alpha_{d} \widetilde{\beta_{d}}+\alpha_{c} \widetilde{\beta_{c}} .
$$

where the phase average over phase $i$ is defined as

$$
\widetilde{\beta_{i}}=\frac{1}{V_{i}} \int_{V_{i}} \beta_{i} d V
$$

The single-field $\beta$ is defined all over the computational grid regardless the nature of the phase that occupies the cells (disperse, continuous or mixture). The VOF methods solve partial differential equations that govern the single-field velocity $\mathbf{u}$, the single-field pressure $p$ and the single-field concentration $c_{j}$ for species $j$. Likewise, the viscosity $\mu$ and density $\rho$ are defined as single-field variables.

The governing equations on the FVM grid are obtained by applying the volume averaging operator, Eq. (11), to the continuity equations, Eq. (1), to the momentum equations, Eq. (3), and to the species conservation laws, Eq. (6), for the continuous and the disperse phases, and then by adding the two phase-averaged equations to form the governing equation for the single-field variable. In the averaging process, the volume averaging operator is applied to the spatial differential operators (gradient and divergence). This operation is not straightforward because integrals and derivatives can not be interchanged in volumes that contain the fluid-fluid interface $\Sigma$. This is achieved using the spatial volume averaging theorems (Whitaker, 1999),

$$
\begin{aligned}
\overline{\nabla \beta_{i}} & =\nabla \overline{\beta_{i}}+\frac{1}{V} \int_{A_{\Sigma}} \beta_{i} \mathbf{n}_{\Sigma} d A, \\
\overline{\nabla \cdot \boldsymbol{\beta}_{i}} & =\nabla \cdot \overline{\boldsymbol{\beta}_{i}}+\frac{1}{V} \int_{A_{\Sigma}} \boldsymbol{\beta}_{i} \cdot \mathbf{n}_{\Sigma} d A,
\end{aligned}
$$

where $A_{\Sigma}=V \cap \Sigma$ is the surface area of the interface within the control volume $V$. The surface integral term in these equations transforms the boundary conditions at the discontinuity $\Sigma$ between the two fluids phases into a body force. For example, the integration of the continuity equations gives rise to the volume source term,

$$
\dot{m}=\frac{1}{V} \int_{A_{\Sigma}} \rho_{i}\left(\mathbf{u}_{i}-\mathbf{w}\right) \cdot \mathbf{n}_{\Sigma} d A,
$$


that describes the rate of phase change within a control volume $V$, i.e. the amount of mass that is transferred from the continuous phase to the disperse phase (and reverse) across the fluid-fluid interface.

In the VOF method, the normal vector $\mathbf{n}_{\Sigma}$ defined on every points of the discontinuity $\Sigma$ is transformed into a mean normal surface that is computed using the gradient of the phase indicator function (Quintard and Whitaker, 1994),

$$
\nabla \alpha_{d}=\frac{1}{V} \int_{A_{\Sigma}} \mathbf{n}_{\Sigma} d A .
$$

Therefore, the average of the normal vector $\mathbf{n}_{\Sigma}$ is understood as

$$
\overline{\mathbf{n}}_{\Sigma}=\frac{\nabla \alpha_{d}}{\left\|\nabla \alpha_{d}\right\|}
$$

$\overline{\mathbf{n}}_{\Sigma}$ is a unit vector defined at the cell centres that describes the mean normal to the fluid-fluid interface in a control volume. In particular, it is used to compute the mean interface curvature.

The single-field equations are obtained following the averaging procedure described above. Here, we present only the final conservation laws. A detailed derivation can be found in Fleckenstein and Bothe (2015). The continuity equation reads

$$
\nabla \cdot \mathbf{u}=\dot{m}\left(\frac{1}{\rho_{d}}-\frac{1}{\rho_{c}}\right) .
$$

Further, the single-field momentum equation can be written as

$$
\frac{\partial \rho \mathbf{u}}{\partial t}+\nabla \cdot(\rho \mathbf{u u})=-\nabla p+\nabla \cdot\left(\mu\left(\nabla \mathbf{u}+\nabla \mathbf{u}^{T}\right)\right)+\rho \mathbf{g}+\mathbf{f}_{\Sigma}
$$

where

$$
\mathbf{f}_{\Sigma}=\frac{1}{V} \int_{A_{\Sigma}} \sigma \kappa \mathbf{n}_{\Sigma} d A
$$

is the mean surface tension force described as a body force. To compute $\mathbf{f}_{\Sigma}$, the curvature within the control volume is approximated by a mean curvature estimated from the normal vector, $\kappa=-\nabla \cdot \overline{\mathbf{n}}_{\Sigma}$ (Brackbill et al., 1992). The surface tension $\sigma$ and the mean curvature are then constant within a control volume, and can be removed from the integral. Therefore, using Eq. (17), $\mathbf{f}_{\Sigma}$ becomes

$$
\mathbf{f}_{\Sigma}=\sigma \kappa \nabla \alpha_{d}
$$

This surface tension force model is known as Continuous Surface Force (CSF) (Brackbill et al., 1992). Since in the VOF method, the fluid interface is represented by an indicator function which changes over a thin region, the estimation of the curvature using the CSF method is inaccurate and can generate non-physical parasitic velocities (Scardovelli and Zaleski, 1999; Popinet, 2018). These spurious currents might be reduced by computing the curvature from a smoothed indicator function $\widehat{\alpha}_{d}$, i.e.

$$
\begin{aligned}
& \kappa=\nabla \cdot\left(\frac{\nabla_{\alpha_{d}}}{\left\|\widehat{\nabla}_{d}\right\|}\right), \\
& \widehat{\alpha}_{d}=\frac{\sum_{f} \alpha_{d_{f}} A_{f}}{\sum_{f} A_{f}},
\end{aligned}
$$

where $\alpha_{d_{f}}$ is the value of $\alpha_{d}$ on the face $f$ of the control volume, obtained by linear interpolation, and $A_{f}$ is the face surface area.

Finally, the volumetric phase equation is

$$
\frac{\partial \alpha_{d}}{\partial t}+\nabla \cdot\left(\alpha_{d} \mathbf{u}\right)+\nabla \cdot\left(\alpha_{d} \alpha_{c} \mathbf{u}_{r}\right)=\frac{\dot{m}}{\rho_{d}},
$$


where $\mathbf{u}_{r}=\widetilde{\mathbf{u}}_{d}-\widetilde{\mathbf{u}}_{c}$ is the relative velocity. This advection equation is exact because its derivation does not imply any assumption (Fleckenstein and Bothe, 2015). Note that the last term on the left-hand side has nonzero value only in the cells containing the fluid/fluid interface. The relative velocity $\mathbf{u}_{r}$ is a consequence of mass and momentum transfer between the phases. However, Fleckenstein and Bothe (2015) showed that $\mathbf{u}_{r}$ may be neglected even in the case of very good solubility (e.g. $\mathrm{CO}_{2}$ in water) in order to simplify Eq. (24).

High resolution differencing schemes such as Flux Corrected Transport (FCT) (Zalesak, 1979) or Normalised Variable Formulation (NVF) (Leonard, 1988) can be used to reduce the smearing of the interface. These methods combined a bounded but diffusive low-order scheme with an unbounded but more accurate higher order scheme. In order to maintain a sharp interface, the amount of downwinding should be maximised, leading to so-called interface capturing schemes such as the Compressive Interface Capturing Scheme for Arbitrary Meshes (CICSAM) (Ubbink and Issa, 1999) and the High Resolution Interface Capturing (HRIC) scheme (Muzaferija et al., 1999). However, the present work is based on the interFoam solver (OpenCFD, 2016), which algebraic advection implementation utilises a FCT method called Multidimensional Universal Limiter with Explicit Solution (MULES) (Weller, 2006). In addition, compression of the interface is performed but, rather than using an interface capturing scheme, an artificial compression term is introduced by replacing $\mathbf{u}_{r}$ in Eq. (24) by a compressive velocity $\mathbf{u}_{c o m p}$, normal to the interface and with an amplitude based on the maximum of the single-field velocity (Rusche, 2002)

$$
\mathbf{u}_{r} \equiv \mathbf{u}_{c o m p}=\overline{\mathbf{n}}_{\Sigma}\left[\min \left(c_{\alpha} \frac{\left|\phi_{f}\right|}{A_{f}}, \max _{f}\left(\frac{\left|\phi_{f}\right|}{A_{f}}\right)\right)\right],
$$

where $c_{\alpha}$ is the compression constant (generally between 0 and 4) and $\phi_{f}$ is the volumetric flux across $f$. In all our simulations, we choose $c_{\alpha}=1.0$.

\subsection{Single-field concentration equation}

Similarly, applying volume-averaging to the mass conservation equation of each component in each phase, and then adding them up, yields to the single-field concentration equation. Deising et al. (2016) proposed a formal derivation of the model, leading to the so-called Continuous Species Transfer (CST) formulation

$$
\frac{\partial c_{j}}{\partial t}+\nabla \cdot \mathbf{F}_{j}+\nabla \cdot \mathbf{J}_{j}=0
$$

where the advective flux is

$$
\mathbf{F}_{j}=\alpha_{d} \widetilde{c_{j, d}} \widetilde{\mathbf{u}_{d}}+\alpha_{c} \widetilde{c_{j, c}} \widetilde{\mathbf{u}_{c}}
$$

and the diffusive flux is

$$
\mathbf{J}_{j}=-\alpha_{d} D_{j, d} \nabla \widetilde{c_{j, d}}-\alpha_{c} D_{j, c} \nabla \widetilde{c_{j, c}} .
$$

In the single-field approach, the fluxes $\mathbf{F}_{j}$ and $\mathbf{J}_{j}$ have to be described in term of single-field variables. Haroun et al. (2010) proposed the following formulation for the diffusion flux

$$
\mathbf{J}_{j}=-D_{j}^{S F} \nabla c_{j}+\boldsymbol{\Phi}_{j}
$$

where $D_{j}^{S F}$ is the single-field diffusion coefficient and $\boldsymbol{\Phi}_{j}$ is the CST flux, defined as

$$
\boldsymbol{\Phi}_{j}=\left(1-H_{j}\right) D_{j}^{S F} \frac{c_{j}}{\alpha_{d}+H_{j} \alpha_{c}} \nabla \alpha_{d}
$$

The debate regarding the best formulation for the single-field diffusion coefficient was initiated in the original work of Haroun et al. (2010) and pursued in Marschall et al. (2012) and Deising et al. (2016). Indeed, Haroun 
et al. (2010) first proposed an arithmetic mean for the diffusion coefficient

$$
D_{j}^{S F} \equiv D_{j}^{a}=\alpha_{d} D_{j, d}+\alpha_{c} D_{j, c}
$$

However, they showed in their own test cases that a harmonic mean was more accurate. Further, Deising et al. (2016) derived the following formulation for the diffusive flux

$$
\mathbf{J}_{j}=-D_{j}^{a} \nabla c_{j}-\alpha_{d} \alpha_{c} \frac{\left(D_{j, d}-D_{j, c}\right)\left(1-H_{j}\right)}{\alpha_{d}+H_{j} \alpha_{c}} \nabla c_{j}+\boldsymbol{\Phi}_{j},
$$

with

$$
\boldsymbol{\Phi}_{j}=\frac{c_{j}}{\alpha_{d}+H_{j} \alpha_{c}}\left(\left(D_{j, d}-H_{j} D_{j, c}\right)-\frac{H_{j}\left(D_{j, d}-D_{j, c}\right)}{\alpha_{d}+H_{j} \alpha_{c}}\right) \nabla \alpha_{d} .
$$

They compared this formulation to the harmonic formulation on several test cases and obtained almost identical results. However, Eqs. (32)-(33) can be drastically simplified by putting everything over the common denominator $\alpha_{d}+H_{j} \alpha_{c}$. Doing so, we can show that this formulation is equivalent to Haroun et al. (2010) formulation (Eqs. (29)-(30)) if the arithmetic mean diffusion is replaced by the equilibrium-based mean diffusion

$$
D_{j}^{S F} \equiv D_{j}^{m}=\frac{\alpha_{d} D_{j, d}+H_{j} \alpha_{c} D_{j, c}}{\alpha_{d}+H_{j} \alpha_{c}}
$$

which states that the diffusion of component $j$ in the interface region is equal to the weighted mean of the phase diffusion coefficients $D_{j, d}$ and $D_{j, c}$ weighted by $\alpha_{d}$ and $H_{j} \alpha_{c}$. This formulation has many advantages over the arithmetic and harmonic formulations proposed by Haroun et al. (2010); Marschall et al. (2012); Deising et al. (2016). It is an equivalent simplified formulation of Eqs. (26)-(33) that ensures continuity of the diffusive flux of dilute species at the interface and that, contrary to the harmonic formulation, can be applied to the case when $D_{j, i} \rightarrow 0$ (for example when the disperse phase is pure).

Finally, the advective flux can be written as (Maes and Soulaine, 2018)

$$
\mathbf{F}_{j}=c_{j} \mathbf{u}+\alpha_{d} \alpha_{c}\left(\widetilde{c_{j, d}}-\widetilde{c_{j, c}}\right) \mathbf{u}_{r}
$$

Since the CST model (Eqs.(26)-(35)) has been derived by volume averaging, it is in principles consistent with the phase advection equation (Eq.1), regardless of the discretization scheme employed. However, Deising et al. (2016) noted that to obtain a fully consistent advection scheme and avoid artificial mass transfer at the interface, it is essential to apply the same discretization scheme to all advected quantities. Following this principle, the CST method has been applied successfully with algebraic VOF methods with FCT (Haroun et al., 2010) or CICSAM (Deising et al., 2018) discretization schemes.

When using high resolution discretization schemes, the relative velocity $\mathbf{u}_{r}$ may be neglected, so the advective flux in the CST model becomes $\mathbf{F}_{j}=c_{j} \mathbf{u}$. However, additional care must be taken when using the CST model with an artificial compressive velocity. Yang et al. (2017) neglected the relative velocity in the species transport equation, and they observed that, when convection dominates diffusion locally near the interface, this formulation generates a large numerical error. They show that in order to capture the discontinuity at the interface accurately, the mesh should be designed so that the diffusion dominates the advection locally. This is described by the local Péclet number $P e_{l o c a l}=\frac{U \delta_{x}}{D}$, and they recommend to use $P e_{l o c a l}<0.5$. However, this restriction cannot be applied for the case when $D_{j, i} \rightarrow 0$ (for example when the disperse phase is pure). Maes and Soulaine (2018) shows that the numerical error is in fact due to inconsistency in the phase and component advection schemes arising from the compression term. It is therefore essential to use the so called Compressive CST (C-CST) (Maes and Soulaine, 
2018) formulation, where the advective flux includes a compressive term

$$
\mathbf{F}_{j}=c_{j} \mathbf{u}+\alpha_{d} \alpha_{c}\left(\widetilde{c_{j, d}}-\widetilde{c_{j, c}}\right) \mathbf{u}_{c o m p}
$$

The phase concentration $\widetilde{c_{j, d}}$ and $\widetilde{c_{j, c}}$ are unknown and need to be expressed as a function of $\alpha_{d}$ and $c_{j}$. In the original C-CST model (Maes and Soulaine, 2018), the phase concentration are assumed to be at equilibrium in the grid blocks that contain the interface, and $\widetilde{c_{j, d}}=\frac{c_{j}}{\alpha_{d}+H_{j} \alpha_{c}}$ and $\widetilde{c_{j, c}}=\frac{H_{j} c_{j}}{\alpha_{d}+H_{j} \alpha_{c}}$. The phase concentration difference was,

$$
\left(\widetilde{c_{j, d}}-\widetilde{c_{j, c}}\right)=\frac{\left(1-H_{j}\right) c_{j}}{\alpha_{d}+H_{j} \alpha_{c}}
$$

In the present paper, we propose a different model based on the computation of the gradient of concentration at the interface. Using the definition of the single-field concentration, we have,

$$
\begin{aligned}
\nabla c_{j} \cdot \nabla \alpha_{d} & =\nabla\left(\alpha_{d} \widetilde{c_{j, d}}+\alpha_{c} \widetilde{c_{j, c}}\right) \cdot \nabla \alpha_{d}, \\
& =\left[\alpha_{d} \nabla \widetilde{c_{j, d}}+\alpha_{c} \nabla \widetilde{c_{j, c}}+\left(\widetilde{c_{j, d}}-\widetilde{c_{j, c}}\right) \nabla \alpha_{d}\right] \cdot \nabla \alpha_{d}, \\
& =\alpha_{d} \nabla \widetilde{c_{j, d}} \cdot \nabla \alpha_{d}+\alpha_{c} \nabla \widetilde{c_{j, c}} \cdot \nabla \alpha_{d}+\left(\widetilde{c_{j, d}}-\widetilde{c_{j, c}}\right)\left\|\nabla \alpha_{d}\right\|^{2} .
\end{aligned}
$$

Although the gradient of the phase concentration across the interface is zero only when the phases are at chemical equilibrium in the cell, the compression may assume that they are in order to avoid additional mass transfer across the interface. Therefore, we assume $\nabla \widetilde{c_{j, d}} \cdot \nabla \alpha_{d}=\nabla \widetilde{c_{j, c}} \cdot \nabla \alpha_{d}=0$. and the phase concentration difference can be written as

$$
\left(\widetilde{c_{j, d}}-\widetilde{c_{j, c}}\right)=\frac{\nabla c_{j} \cdot \nabla \alpha_{d}}{\left\|\nabla \alpha_{d}\right\|^{2}}
$$

In the rest of the paper, this model is referred to as the normal C-CST model while the model using Eq. (37) is referred to as the original C-CST. The two C-CST models are compared for a 1D test case with infinite Péclet number in section 3. In particular, we observe that the local mass transfer between the phase generated by the compression is zero for the normal C-CST model but not for the original C-CST model. This is an essential property for computing interface mass transfer and local volume change.

\subsection{Interface mass transfer}

The VOF approach with local volume change requires the computation of the rate of mass transfer, $\dot{m}$, at the gas/liquid interface. Indeed, $\dot{m}$ appears both in the continuity equation, Eq. (19), and in the phase conservation law, Eq. (24). By definition, the rate of mass transfer, responsible for the local phase volume change, results from the sum of the rate of transfer, $\dot{m}_{j}$, of all the species that cross the interface, i.e. all the species except for the one labelled $n$ that remains always in the continuous phase. Hence, the rate of mass transfer writes

$$
\dot{m}=\sum_{1 \leq j<n} \dot{m}_{j},
$$

where the rate of species transfer is defined in each control volume $V$ that contains part of the interface $\Sigma$ by integrating the flux condition Eq. (9) over the surface of the interface. We have,

$$
\dot{m}_{j}=\dot{m}_{j, i}=\frac{1}{V} \int_{A_{\Sigma}}\left(c_{j, i}\left(\mathbf{u}_{i}-\mathbf{w}\right)-D_{j, i} \nabla c_{j, i}\right) \cdot \mathbf{n}_{\Sigma} d A
$$

This integral involves variables defined in the continuous physical space and needs to be formulated in terms of grid-based variables to be used in the VOF method. In a control volume, the concentration at the interface is assumed to verify $c_{j, i_{\Sigma}} \approx \widetilde{c_{j, i}}$ and $\nabla c_{j, i_{\Sigma}} \approx \nabla \widetilde{c_{j, i}}$ (Soulaine et al., 2011). Because, $\widetilde{c_{j, i}}$ is a volume averaged quantity 
over the control volume $V$, it has a constant value all along $A_{\Sigma}$ and can be pull out the surface integral. Finally, using Eq. (16) and Eq. (17), we obtain

$$
\begin{aligned}
\dot{m}_{j, i} & =\frac{1}{V} \int_{A_{\Sigma}}\left(\widetilde{c_{j, i}}\left(\mathbf{u}_{i}-\mathbf{w}\right)-D_{j, i} \nabla \widetilde{c_{j, i}}\right) \cdot \mathbf{n}_{\Sigma} d A, \\
& =\widetilde{c_{j, i}} \frac{1}{V} \int_{A_{\Sigma}}\left(\mathbf{u}_{i}-\mathbf{w}\right) \cdot \mathbf{n}_{\Sigma} d A-D_{j, i} \nabla \widetilde{c_{j, i}} \cdot \frac{1}{V} \int_{A_{\Sigma}} \mathbf{n}_{\Sigma} d A, \\
& =\frac{\widetilde{c_{j, i}}}{\rho_{i}} \frac{1}{V} \int_{A_{\Sigma}} \rho_{i}\left(\mathbf{u}_{i}-\mathbf{w}\right) \cdot \mathbf{n}_{\Sigma} d A-D_{j, i} \nabla \widetilde{c_{j, i}} \cdot \frac{1}{V} \int_{A_{\Sigma}} \mathbf{n}_{\Sigma} d A, \\
& =\frac{\widetilde{c_{j, i}}}{\rho_{i}} \dot{m}-D_{j, i} \nabla \widetilde{c_{j, i}} \cdot \nabla \alpha_{d} .
\end{aligned}
$$

Eq. (42) cannot be used in its present form since it depends on $\widetilde{c_{j, i}}$ that is not computed in the VOF method. It is recast into a form that depends only on the single-field variables using the sum of the species transfer rate on each side of the interface weighted by a fluid volume fraction. We have,

$$
\begin{aligned}
\dot{m}_{j} & =\alpha_{d} \dot{m}_{j, d}+\alpha_{c} \dot{m}_{j, c}, \\
& =\alpha_{d}\left(\widetilde{\frac{c_{j, d}}{\rho_{d}}} \dot{m}-D_{j, d} \nabla \widetilde{c_{j, d}} \cdot \nabla \alpha_{d}\right)+\alpha_{c}\left(\widetilde{\frac{c_{j, c}}{\rho_{c}}} \dot{m}-D_{j, c} \nabla \widetilde{c_{j, c}} \cdot \nabla \alpha_{d}\right), \\
& =\left(\alpha_{d} \frac{\widetilde{c_{j, d}}}{\rho_{d}}+\alpha_{c} \frac{\widetilde{c_{j, c}}}{\rho_{c}}\right) \dot{m}-\left(\alpha_{d} D_{j, d} \nabla \widetilde{c_{j, d}}+\alpha_{c} D_{j, c} \nabla \widetilde{c_{j, c}}\right) \cdot \nabla \alpha_{d}, \\
& =X_{j} \dot{m}+\mathbf{J}_{j} \cdot \nabla \alpha_{d},
\end{aligned}
$$

where $X_{j}=\left(\alpha_{d} \frac{\widetilde{c j, d}}{\rho_{d}}+\alpha_{c} \frac{\widetilde{c_{j, c}}}{\rho_{c}}\right)$, and where the diffusion flux was simplified using Eqs. (28) and (29). Using Eq. (40) along with Eq. (43) we obtain

$$
\dot{m}=\sum_{1 \leq j<n} X_{j} \dot{m}+\sum_{1 \leq j<n} \mathbf{J}_{j} \cdot \nabla \alpha_{d} .
$$

$$
\sum_{1 \leq j<n} X_{j}=\alpha_{d}+\alpha_{c}\left(1-\frac{\widetilde{c_{n, c}}}{\rho_{c}}\right) \approx \alpha_{d}
$$

Therefore, using Eq. (29), the total mass transfer in a control volume that includes interfacial area is calculated as

$$
\dot{m}=-\frac{\sum_{1 \leq j<n}\left(D_{j}^{m} \nabla c_{j}-\boldsymbol{\Phi}_{j}\right)}{1-\alpha_{d}} \cdot \nabla \alpha_{d}
$$

This closes Eq. (24) and the entire system.

\subsection{Numerical implementation}

The numerical method has been implemented in our OpenFOAM ${ }^{\circledR}$-based reactive transport solver (OpenCFD, 2016). The standard VOF solver of OpenFOAM ${ }^{\circledR}$, so-called interFoam has been extended for this purpose into another solver called interTransferFoam. The full solution procedure is presented in Fig. 2. interFoam solves the system formed by Eq. (19), (24) and (20) on a collocated Eulerian grid. A pressure equation is obtained by combining the continuity (Eq. (19)) and momentum (Eq. (20)) equations. The system is then solved with a predictor-corrector strategy based on the Pressure Implicit Splitting Operator (PISO) algorithm (Issa et al., 1985). Three iterations of the PISO loop are used to stabilise the system. An explicit formulation is used to treat the coupling between the phase distribution equation (Eq. (24)) and the pressure equation. This imposes a limit on the 


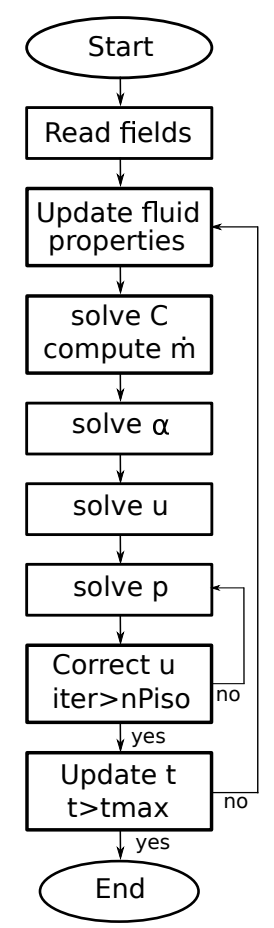

Figure 2: Full solution procedure for interTransferFoam.

time-step size by introducing a capillary wave time scale described by the Brackbill conditions (Brackbill et al., 1992).

In interTransferFoam, the concentration equation (Eq. (26)) is solved sequentially before the phase conservation. The interfacial mass transfer (Eq. (46)) is then computed and re-injected in the continuity (Eq. (19)) and phase equations (Eq. (24)). The space discretization of the convection terms is performed using the second-order vanLeer scheme (van Leer, 1974). For the compression terms, the interpolation of $\alpha_{d} \alpha_{c}$ is performed using the interfaceCompression scheme (OpenCFD, 2016). The diffusion term $\nabla .\left(D_{j}^{m} \nabla c_{j}\right)$ is discretized using the Gauss linear limited corrected scheme, which is second order and conservative. The discretization of the CST flux is performed using the Gauss linear scheme.

For the computation of the mass transfer $\dot{m}$, we define

$$
\boldsymbol{\Phi}_{D}=\frac{\sum_{1 \leq j<n} D_{j}^{m} \nabla c_{j}-\boldsymbol{\Phi}_{j}}{1-\alpha_{d}}
$$

and then we use $\boldsymbol{\Phi}_{D} \cdot \nabla \alpha_{d}=\nabla \cdot\left(\boldsymbol{\Phi}_{D} \alpha_{d}\right)-\alpha_{d} \nabla \cdot \boldsymbol{\Phi}_{D}$. The discretization of $\boldsymbol{\Phi}_{D} \alpha_{d}$ is performed using upwinding in the direction of $\nabla \alpha_{d}$ in order to prevent negative $\alpha_{d}$. This is only first-order accurate (see Section 3), but all second-order discretization schemes available in OpenFOAM ${ }^{\circledR}$ have shown strong instabilities.

\subsection{Dimensionless analysis and large-scale post-processing}

In this section, we describe the dimensionless numbers used to analyse the simulation results in terms of bulk-averaged properties.

For two-phase flow, the relative importance of inertia and viscous forces is quantified using the Reynolds number Re, while the importance of gravity and surface tension force is characterised using the Eötvös Eo and Morton Mo numbers,

$$
\operatorname{Re}=\frac{\rho_{c} U L}{\mu_{c}}, \quad \text { Eo }=\frac{\Delta \rho g L^{2}}{\sigma}, \quad \text { Mo }=\frac{g \mu_{c}^{4} \Delta \rho}{\rho_{c}^{2} \sigma^{3}},
$$


where $U$ and $L$ are reference velocity and length, respectively.

Similarly, the relative importance of viscous and molecular diffusivity is quantified using the Schmidt number Sc, while the Péclet number $\mathrm{Pe}=\mathrm{Sc} \times \mathrm{Re}$ is equal to the ratio of advective to diffusive transport

$$
\mathrm{Sc}=\frac{\mu_{c}}{\rho_{c} D_{j, c}}, \quad \mathrm{Pe}=\frac{U L}{D_{j, c}} .
$$

A common engineering approach for the modelling of species transfer across interfaces is to introduce a species transfer coefficient $k_{j}$

$$
\int_{\Omega} \dot{m}_{j} d V=k_{j} A_{\Sigma}\left(H_{j} c_{j, d, \infty}-c_{j, c, \infty}\right),
$$

where $c_{j, i, \infty}$ is the bulk average concentration of $j$ in phase $i$. This leads to the definition of the Sherwood number $\mathrm{Sh}$, which quantifies the ratio of interface mass transfer rate to the molecular diffusion rate

$$
\mathrm{Sh}=\frac{k_{j} L}{D_{j, c}} .
$$

Establishing accurate Sherwood correlation as a function of the other dimensionless groups (Reynolds, Schmidt, Péclet) is one of the most investigated engineering question in bubble column reactors (Oellrich et al., 1973; Clift et al., 1978; Takemura and Yabe, 1998).

\section{Verification}

\subsection{Two-phase transport in a $1 D$ tube at infinite Péclet number}

The objective of this test case is to observe and quantify the artificial mass transfer that arises at the interface when compression is not applied consistently for the phase volume fraction and species concentration. For this, four numerical implementations are compared in OpenFOAM ${ }^{\circledR}$ (OpenCFD, 2016). First, no interface compression is used. The advection is performed using the FCT method implemented in MULES (Weller, 2006), but the compression is cancelled by setting $c_{\alpha}=0$. The species concentration equation is modelled using the standard CST method. This implementation is labelled VOF-FCT0-S-CST. Secondly, we used compression of the interface for the phase fraction $\left(c_{\alpha}=1\right)$, but not for the species concentration. This implementation is labelled VOF-FCT1S-CST. Thirdly, we use compression of the interface for the phase fraction and for the species concentration, using the original C-CST method (Eqs. (36) and (37)). This implementation is labelled VOF-FCT1-OC-CST. Finally, we use compression of the interface for the phase fraction and for the species concentration, using the normal C-CST method (Eqs. (36) and (39)). This implementation is labelled VOF-FCT1-NC-CST.

To compare the four implementations in terms of artificial mass transfer, we consider a 1D tube of length $200 \mu \mathrm{m}$. The tube is initially filled with the disperse phase $\left(\alpha_{d}=1\right)$ from $\mathrm{x}=0$ to $40 \mu \mathrm{m}$ and the continuous phase $\left(\alpha_{d}=0\right)$ from $\mathrm{x}=40$ to $200 \mu \mathrm{m}$. The concentration of a species $A$ is set to $1 \mathrm{~kg} / \mathrm{m}^{3}$ in the disperse phase and 0 in the continuous phase. The Henry coefficient $H_{A}$ is assumed to be equal to 0.5 , so that the system is not at equilibrium. The velocity is constant $U=0.05 \mathrm{~m} / \mathrm{s}$ in the domain. In addition, we assume that $D_{A, d}=D_{A, c}=0$ (infinite Pe). Therefore, there is no transfer between the phase and the analytical solution for both phase fraction and species concentration is a sharp front moving at speed $U$.

The simulation are performed on a regular grid with resolution $\Delta x=1 \mu \mathrm{m}$ and with a constant time-step $\Delta t=10^{-7} \mathrm{~s}$. Fig. 3 shows the phase fraction and concentration profiles at different time for the four different implementations and Fig. 4 shows the evolution of the fraction of component $A$ in the continuous phase. For the case with no compression (VOF-FCT0-S-CST), the interface is slightly smeared and spans over seven cells (Fig. 3a). However, no artificial mass transfer is observed (Fig. 4). The advection operators for phase fraction and species concentration are fully consistent. For the case where compression is applied to the phase fraction but 

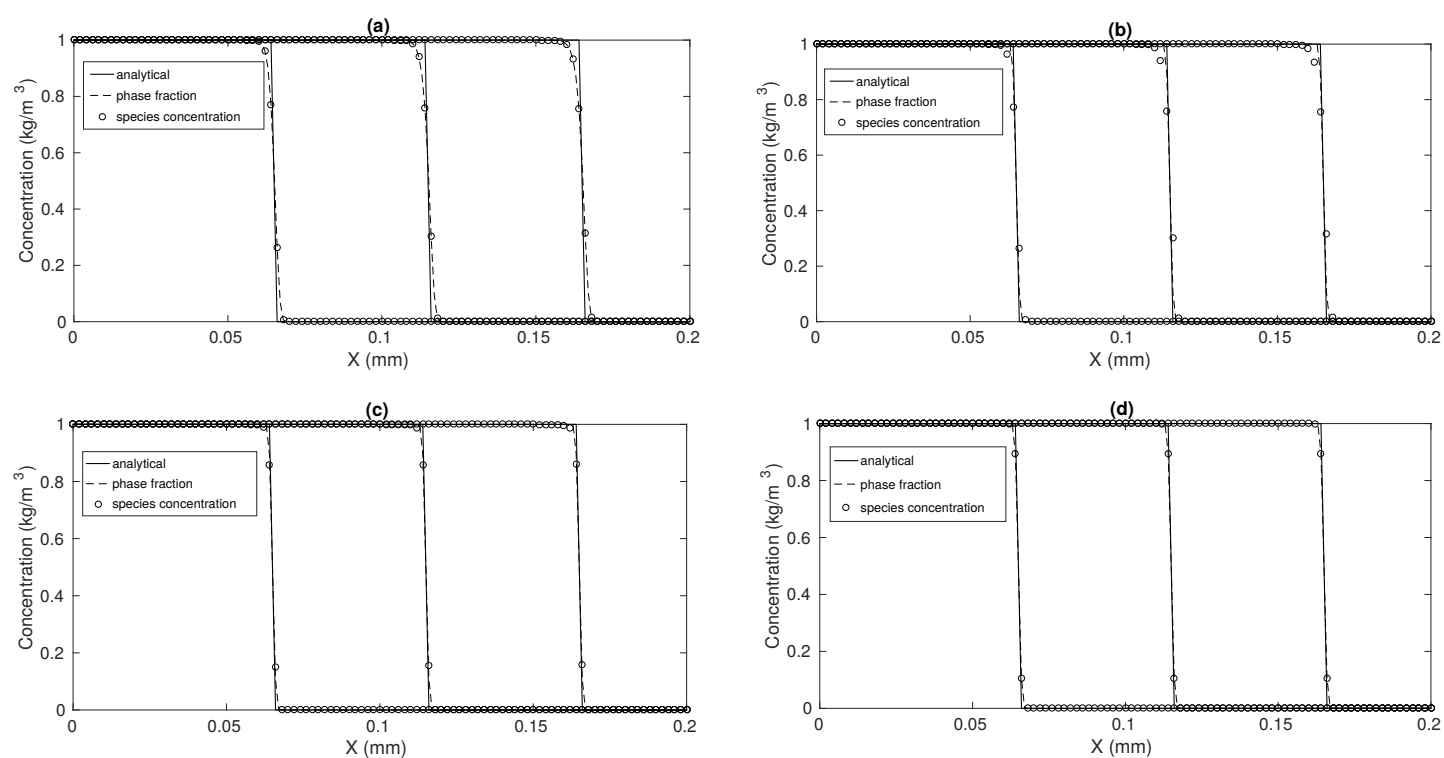

Figure 3: Concentration profile during two-phase transport in a 1D tube at different time obtained using (a) VOF-FCT0-S-CST, (b) VOFFCT1-S-CST, (c) VOF-FCT1-OC-CST and (d) VOF-FCT1-NC-CST.

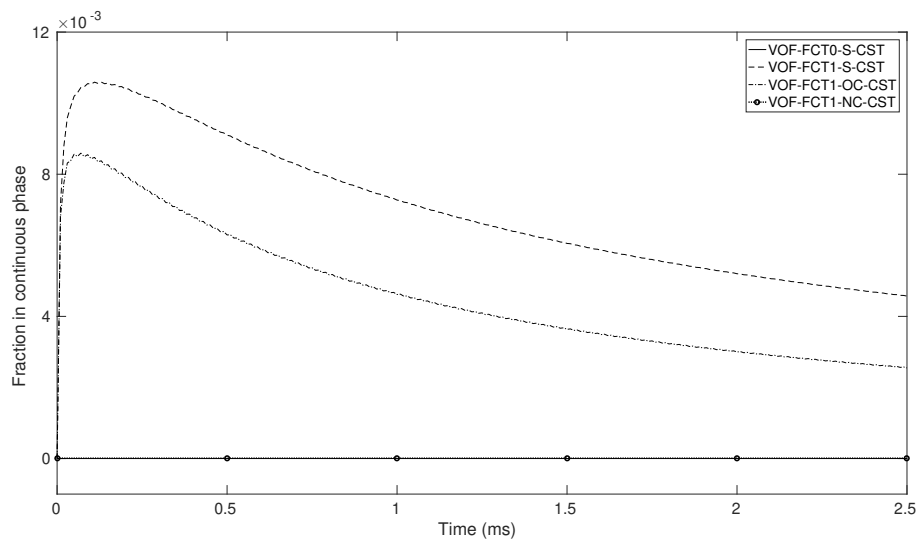

Figure 4: Evolution of the average concentration of component $A$ in the continuous phase during two-phase transport in a 1D tube using the four different implementation of the VOF-CST methods. When compression is applied to the interface, the standard CST and the original C-CST model generate an erroneous transfer between the phases, while for the normal C-CST model, the concentration of $A$ in the continuous phase remains zero.

not to the species concentration (VOF-FCT1-S-CST), the interface is sharper, spanning over three cells, but the species concentration is diffuse (Fig. 3b). The fraction of $A$ in the continuous phase does not remain equal to zero, but reaches a maximum value of 0.01 at $\mathrm{t}=0.15 \mathrm{~ms}$. The erroneous mass transfer between the phases is maximum at $\mathrm{t}=0$, with value equal to $0.012 \mathrm{~kg} / \mathrm{m}^{2} / \mathrm{s}$. This error corresponds to a diffusion coefficient of $1.2 \times 10^{-6} \mathrm{~m}^{2} / \mathrm{s}$. The advection operators for phase fraction and species concentration are not consistent. Next, when the original C-CST method is applied to the species concentration (VOF-FCT1-OC-CST), the species concentration is slightly less diffuse, but the problem is not fully corrected (Fig. 3c). Again, the fraction of $A$ in the continuous phase does not remain equal to zero, but reaches a maximum value of $8 \times 10^{-3}$ at $\mathrm{t}=0.1 \mathrm{~ms}$. The erroneous mass transfer between the phases is also maximum at $\mathrm{t}=0$, with the same value of $0.012 \mathrm{~kg} / \mathrm{m}^{2} / \mathrm{s}$. In the original C-CST model, the phase concentration at the interface are obtained by assuming local equilibrium between the phases. However, when the phase concentrations deviate strongly from equilibrium, artificial mass transfer between the phases 
arises. The original C-CST method is only consistent with artificial compression when phase concentrations at the interface are at equilibrium. In the present case, equilibrium is never reached since Pe is infinite. Finally, when the normal C-CST method is applied (VOF-FCT1-NC-CST), both phase fraction and species concentration are sharp, spanning over three cells (Fig. 3d), and no artificial mass transfer is observed. This is because the normal C-CST model is built so that no interface transfer is generated by the compression (Eq. (38)). This is an essential result when computing the interface mass transfer and the local volume change. The advection operators for phase fraction and species concentration are fully consistent. In the rest of the paper, we will use compression of the interface with the normal C-CST method.

\subsection{Dissolution of a single-component gas phase into an immiscible liquid solvent in a semi-infinite tube}

The objective of this test case is to validate the computation of the interface mass transfer and the local volume change by comparison with a system where an analytical solution exists. For this, we consider the case where the disperse phase is a single-component gas with $\rho_{d}=1 \mathrm{~kg} / \mathrm{m}^{3}$ and the continuous phase is a binary liquid mixture with $\rho_{c}=1000 \mathrm{~kg} / \mathrm{m}^{3}$. The species $A$ of the disperse phase is a dilute component in phase $c$, with $H_{A}=0.5$ and $D_{A, c}=10^{-6} \mathrm{~m}^{2} / \mathrm{s}$. The second species is a solvent that is not soluble in $d$. The domain is a 1D semi-infinite tube (Fig. 5). The gas/liquid interface is initially positioned at a distance $l_{0}=0.5 \mathrm{~mm}$ from the left boundary. Initially, the concentration of dilute species in the liquid is equal to zero.

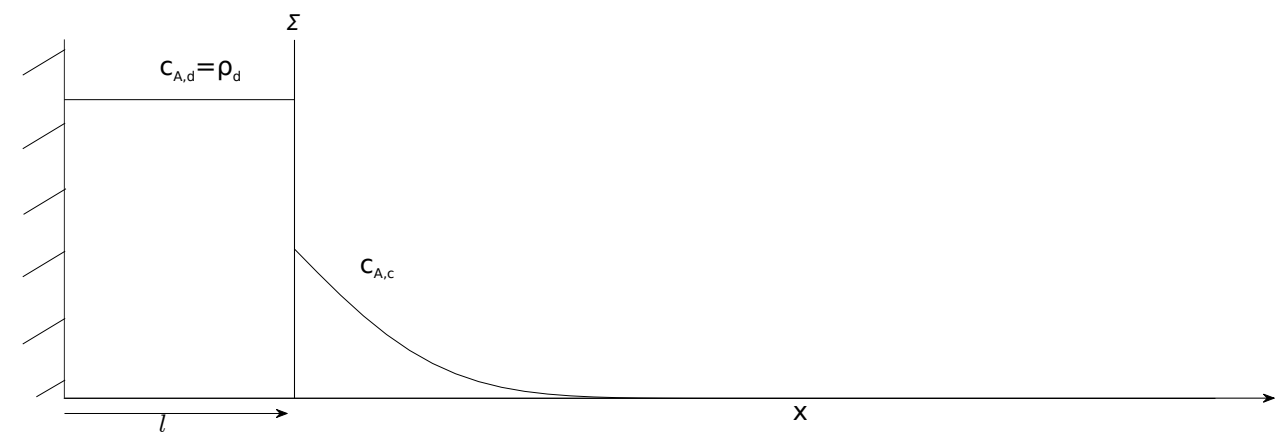

Figure 5: Set-up for dissolution of a single-component gas phase into an immiscible liquid solvent in a semi-infinite tube

Due to incompressibility, the velocity in each phase is constant, and equal to zero in the gas phase. Mass conservation at the interface (Eq. (2)) gives

$$
u_{c}=\frac{\rho_{c}-\rho_{d}}{\rho_{c}} w \text { at } x=l
$$

Therefore, the mass conservation of the dilute component in $c$ writes

$$
\begin{aligned}
& \frac{\partial c_{A . c}}{\partial t}+\frac{\rho_{c}-\rho_{d}}{\rho_{c}} w \frac{\partial c_{A, c}}{\partial x}=D_{A, c} \frac{\partial^{2} c_{A, c}}{\partial x^{2}} \quad \text { for } x>l, \\
& c_{A, c}(x, 0)=0 \quad \text { for } x>l \text {, } \\
& c_{A, c}(l, t)=H_{A} \rho_{d} \quad \text { for } t>0, \\
& \frac{d l}{d t}=w={\frac{D_{A, c}}{\rho_{d}}}_{\frac{\partial c_{A, c}}{\partial x}}{ }_{\mid x=l} \quad \text { for } t>0
\end{aligned}
$$


and the error for concentration and interface position

$$
\begin{aligned}
& \operatorname{Err}\left(c\left(\Delta x^{*}\right)\right)=\max _{t}\left(\sqrt{\int\left(\frac{c\left(\Delta x^{*}, t\right)-c\left(\Delta x^{*} \rightarrow 0, t\right)}{\rho_{d}}\right)^{2}} d t\right), \\
& \operatorname{Err}\left(l\left(\Delta x^{*}\right)\right)=\max _{t}\left(\frac{\left|l\left(\Delta x^{*}, t\right)-l\left(\Delta x^{*} \rightarrow 0, t\right)\right|}{l_{0}}\right) .
\end{aligned}
$$

Fig. 6 shows the evolution of the convergence errors with grid resolution. All simulations are performed with a time-step of $0.2 \mu \mathrm{s}$, for which they have all converged in time. We observe that both measures show first-order

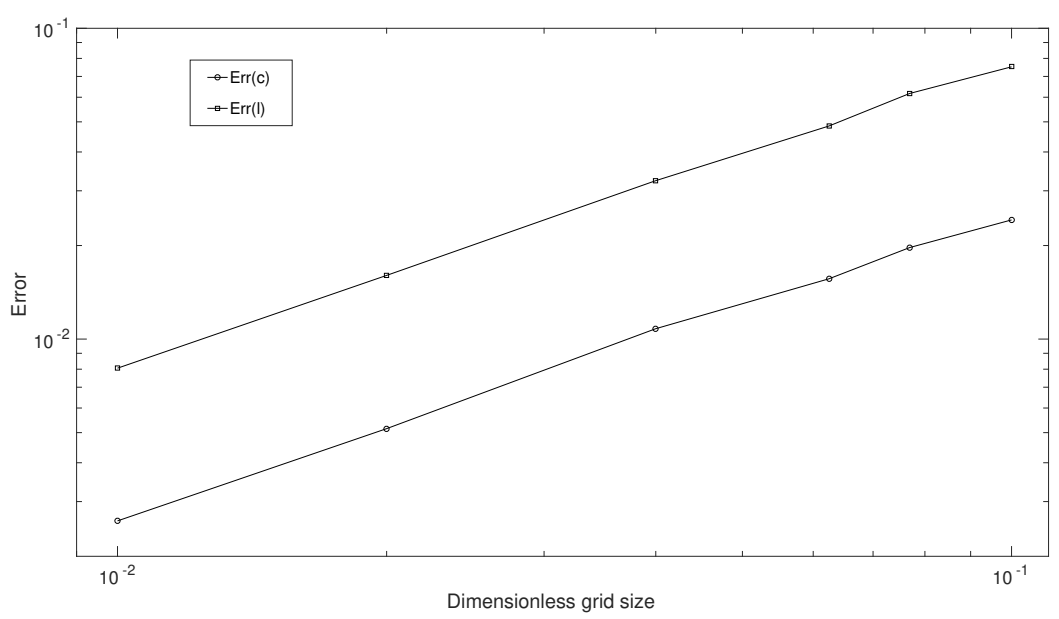

Figure 6: Evolution of convergence of concentration and interface position during dissolution of a single-component gas phase into an immiscible liquid solvent in a semi-infinite tube. Both measures show first-order convergence.

convergence. Although the CST model itself is second-order accurate, the discretization of the phase transfer flux (Eq. 46) is performed using upwinding to avoid negative volume fraction. We have tested the second-order accurate schemes included in OpenFOAM but they all show strong instabilities.

Fig. 7 show a comparison of analytical and numerical simulations for grid resolution $\Delta x^{*}=0.01$. We observe a very good agreement between the simulation and the exact solution. Both convergence errors (Eq. (56)) are smaller than 0.01 .

\subsection{Growth of binary gas in infinite cylinder}

The objective of this test case is to validate the computation of the local volume change when two species are present in the gas phase. For this, we consider an infinite cylinder of radius $R=1 \mathrm{~mm}$. The initial radius of the 

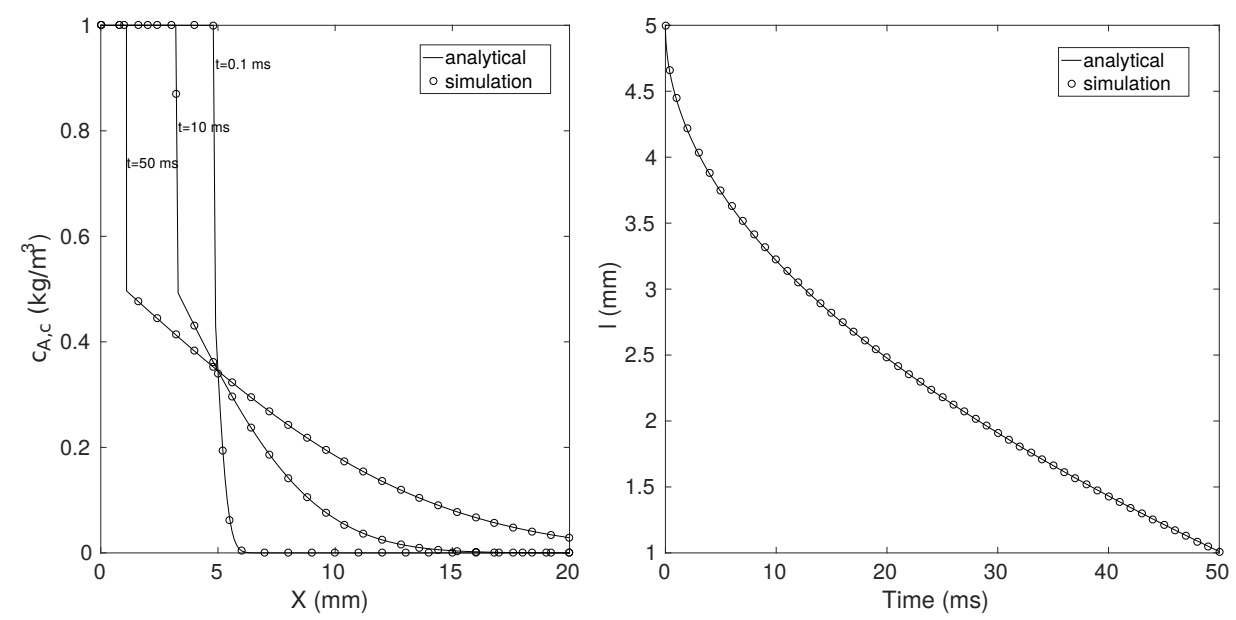

Figure 7: Comparison of analytical and numerical simulation for (a) evolution of species concentration and (b) evolution of interface position during dissolution of a single-component gas phase into an immiscible liquid solvent in a semi-infinite tube. Both concentration and interface position errors are smaller than 0.01 .

dispersed gas phase is $R_{0}=0.5 \mathrm{~mm}$, so that the domain is filled with gas for $0 \leq r<R_{d}$ and with the continuous liquid phase for $R_{d}<r<R$ (Fig. 8). The fluid properties are summarised in Table 1 .

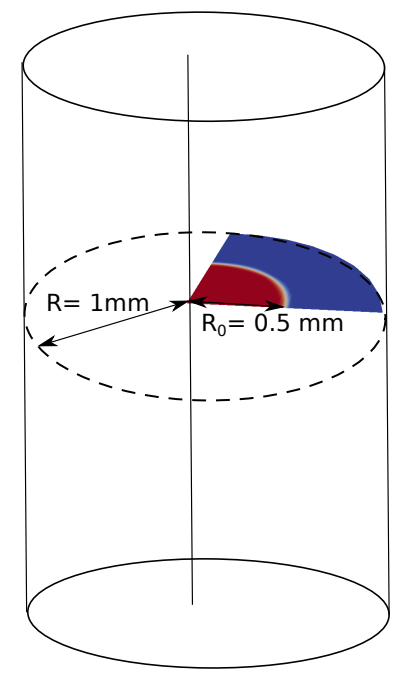

Figure 8: Set-up for growth of binary gas in infinite cylinder.

\begin{tabular}{cccc} 
& $\begin{array}{c}\text { Density } \\
\left(\mathrm{kg} / \mathrm{m}^{3}\right)\end{array}$ & $\begin{array}{c}\text { Dynamic viscosity } \\
(\text { Pa.s })\end{array}$ & $\begin{array}{c}\text { Interfacial tension } \\
(\mathrm{mN} / \mathrm{m})\end{array}$ \\
\hline Gas & 1 & $1.8 \times 10^{-5}$ & 60 \\
Liquid & 1000 & $1 \times 10^{-3}$ & \\
\hline
\end{tabular}

Table 1: Fluid properties for mass transfer during growth of binary gas in infinite cylinder

We consider that the gas is a binary mixture $(\mathrm{A}+\mathrm{B})$. Initially, the gas phase is composed at $100 \%$ of $\mathrm{B}\left(c_{A, d}=0\right.$, $\left.c_{B, d}=\rho_{d}\right)$. We assume that $\mathrm{A}$ is only weakly soluble $\left(H_{A}=0.01\right)$ and $\mathrm{B}$ is not soluble $\left(H_{B}=0\right)$ in liquid, so that the liquid phase is a binary mixture of $\mathrm{A}$ and a solvent that is not soluble in the gas phase. The diffusion coefficient are $D_{A, d}=D_{B, d}=10^{-4} \mathrm{~m}^{2} / \mathrm{s}$ and $D_{A, c}=D_{B, c}=10^{-6} \mathrm{~m}^{2} / \mathrm{s}$. At the outside boundary of the system, the pressure 
remains constant, and the concentration of $\mathrm{A}$ is equal to $H_{A} \rho_{d}$. Due to the symmetry of the problem, the velocity and concentration only depends on the radius $r$, and the motion induced in the liquid phase is also radial,

$$
\mathbf{w}=w(t) \vec{e}_{r}, \mathbf{u}_{c}=u_{c}(r, t) \vec{e}_{r}
$$

Since $\rho_{d}<<\rho_{c}$, mass conservation at the interface (Eq. (2)) gives

$$
u_{c}\left(R_{d}, t\right)=w(t)
$$

Due to the large diffusion ratio between the gas and liquid phases, the species concentration in the gas phase can be considered uniform at the time scale of the process. Since the total mass of B is a constant, the concentration of $\mathrm{A}$ in the gas phase satisfies

$$
c_{A, d}=\rho_{d}\left(1-\left(\frac{R_{0}}{R_{d}}\right)^{2}\right) .
$$

Mass conservation of the dilute component in the liquid phase writes

$$
\begin{array}{ll}
\frac{\partial c_{A, c}}{\partial t}+u_{c} \frac{\partial c_{A, c}}{\partial r}=\frac{D_{A, c}}{r} \frac{\partial}{\partial r}\left(r \frac{\partial c_{A, c}}{\partial r}\right) & \text { for } r>R_{d}, \\
u_{c}(r, t)=\frac{R_{d}}{r} w(t) & \text { for } r>R_{d}, \\
c_{A, c}\left(R_{d}, t\right)=H_{A} \rho_{d}\left(1-\left(\frac{R_{0}}{R_{d}}\right)^{2}\right), c_{A, c}(R, t)=H_{A} \rho_{d} & \text { for } t>0, \\
\frac{d R_{d}}{d t}=w=\frac{D_{A, c}}{\rho_{d}} \frac{\partial c_{A, c}}{\partial r}{ }_{\mid r=R_{d}} & \text { for } t>0 .
\end{array}
$$

Eq. (60) can be written in its dimensionless form using

$$
r^{*}=\frac{r}{R}, R_{d}^{*}=\frac{R_{d}}{R}, t^{*}=\frac{t}{\tau}, c^{*}=\frac{c_{A, c}}{H_{A} \rho_{d}},
$$

where asterisks denote dimensionless variables and

$$
\tau=\frac{R^{2}}{H_{A} D_{A, c}} .
$$

The time-scale $\tau$ corresponds to the time it takes a mass $\pi \rho_{d} R^{2} L$ in a cylinder of radius $\mathrm{R}$ and length $\mathrm{L}$ to diffuse out of the system if the mass flux density is $D_{A, c} L \pi H_{A} \rho_{d}$. For $D_{A, c}=10^{-6} \mathrm{~m}^{2} / \mathrm{s}, \tau=100 \mathrm{~s}$. Substituting into Eq. (60) yields

$$
\begin{array}{ll}
H_{A}\left(\frac{\partial c^{*}}{\partial t^{*}}+w^{*} \frac{R_{d}^{*}}{r^{*}} \frac{\partial c^{*}}{\partial r^{*}}\right)=\frac{1}{r^{*}} \frac{\partial}{\partial r^{*}}\left(r^{*} \frac{\partial c^{*}}{\partial r^{*}}\right) & \text { for } r^{*}>R_{d}^{*}, \\
c^{*}\left(R_{d}^{*}, t^{*}\right)=1-\left(\frac{R_{d}^{*}(0)}{R_{d}^{*}}\right)^{2}, c^{*}\left(1, t^{*}\right)=1 & \text { for } t^{*}>0, \\
\frac{d R_{d}^{*}}{d t^{*}}=w^{*}=\frac{\partial c^{*}}{\partial r^{*}} \mid r^{*}=R_{d}^{*} & \text { for } t^{*}>0
\end{array}
$$

Since $H_{A}<<1$, the concentration of $A$ in the liquid phase can be assumed at equilibrium at the time-scale of the 
motion of the interface. Therefore, Eq. (63) becomes

$$
\begin{array}{ll}
\frac{1}{r^{*}} \frac{\partial}{\partial r^{*}}\left(r^{*} \frac{\partial c^{*}}{\partial r^{*}}\right)=0 & \text { for } r^{*}>R_{d}^{*}, \\
c^{*}\left(R_{d}^{*}, t^{*}\right)=1-\left(\frac{R_{d}^{*}(0)}{R_{d}^{*}}\right)^{2}, c^{*}\left(1, t^{*}\right)=1 & \text { for } t^{*}>0, \\
\frac{d R_{d}^{*}}{d t^{*}}=w^{*}=\frac{\partial c^{*}}{\partial r^{*}} \mid r^{*}=R_{d}^{*} & \text { for } t^{*}>0
\end{array}
$$

An analytical solution for Eq. (64) writes

$$
\begin{aligned}
& c_{A, c}(r, t)=H_{A} \rho_{d}\left(1-\left(\frac{R_{0}}{R_{d}}\right)^{2} \frac{\ln (r / R)}{\ln \left(R_{d} / R\right)}\right), \text { for } r>R_{d}, \\
& \frac{1}{4}\left(R_{d}^{4} \ln \left(\frac{R_{d}}{R}\right)-R_{0}^{4} \ln \left(\frac{R_{0}}{R}\right)\right)-\frac{1}{16}\left(R_{d}^{4}-R_{0}^{4}\right)=-R_{0}^{2} H_{A} D_{A, c} t, \text { for } t>0 .
\end{aligned}
$$

and the error for concentration and gas radius

$$
\begin{aligned}
& \operatorname{Err}\left(c\left(\Delta x^{*}\right)\right)=\max _{t}\left(\sqrt{\int\left(\frac{c_{A, c}\left(\Delta x^{*}, t\right)-c_{A, c}\left(\Delta x^{*} \rightarrow 0, t\right)}{H_{A} \rho_{d}}\right)^{2}} d t\right) \\
& \operatorname{Err}\left(R_{d}\left(\Delta x^{*}\right)\right)=\max _{t}\left(\frac{\left|R_{d}\left(\Delta x^{*}, t\right)-R_{d}\left(\Delta x^{*} \rightarrow 0, t\right)\right|}{R_{d}(0)}\right) .
\end{aligned}
$$

The domain is first meshed with a uniform Cartesian grid, then all cells containing solid are removed and replaced by rectangular and triangular cells that match the solid boundaries, using the OpenFOAM ${ }^{\circledR}$ utilities snappyHexMesh (OpenCFD, 2016). Fig. 9 shows the evolution of the convergence errors with grid resolution. All simulations are performed with a time-step of $0.1 \mu \mathrm{s}$, for which they have all converged in time. Again, we ob-

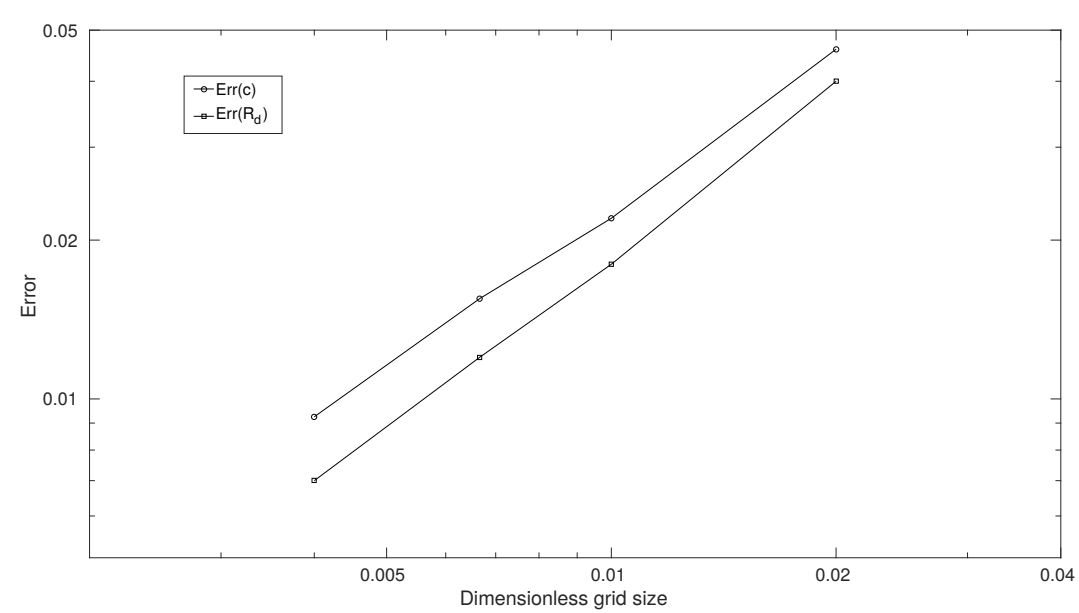

Figure 9: Evolution of convergence of concentration and gas radius during growth of binary gas in infinite cylinder. Both measures show first-order convergence. 
Figure 10: Evolution of dimensionless concentration of component A (colour map) and of gas-liquid interface (white curve) during growth of binary gas in infinite cylinder.

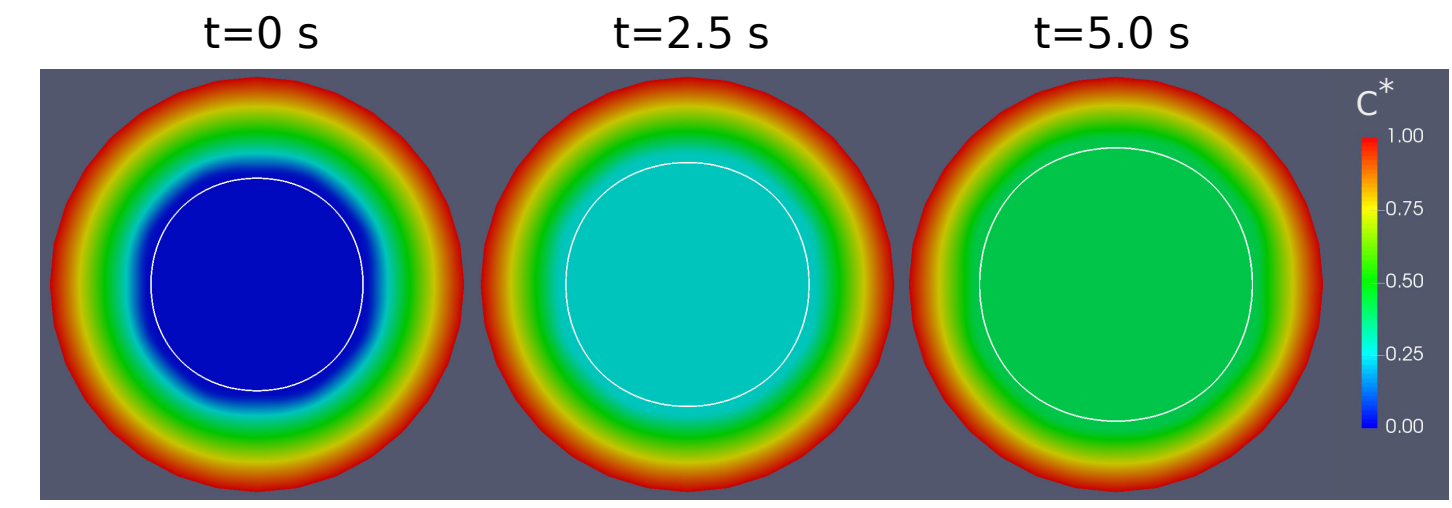

serve that both measures show first-order convergence, due to the first-order upwinding discretization of the phase transfer flux (Eq. 46).

Fig. 10 shows the evolution of the dimensionless concentration of component $\mathrm{A}$ and of the gas-liquid interface, for a grid resolution of $\Delta x^{*}=4 \times 10^{-3}$, obtain using snappyHexMesh with an initial Cartesian grid of $250 \times 250$. The final grid contains 46801 cells.

We observe that the volume of gas grows as component A diffuses across the interface. Fig. 11 shows a comparison between numerical and analytical solution for the dimensionless concentration and radius. The observed errors $\left(9 \times 10^{-3}\right.$ for concentration, $7 \times 10^{-3}$ for radius) are due to the difficulty of maintaining a sharp interface. In addition, we observe that the curvature of the interface is not perfectly circular (Fig. 10). In fact, it oscillates slightly during the simulation due to parasitic currents. In our work, the impact of these spurious currents is only limited and smoothing the indicator function prior to the computation of interface curvature is enough to limit them. To increase robustness and accuracy, various techniques to reduce them further can be considered, such as sharpening of indicator function prior to surface tension force computation (Francois et al., 2006), filtering of capillary force (Raeini et al., 2012) or coupled VOF level-set method (Albadawi et al., 2013), but are outside the scope of this work.
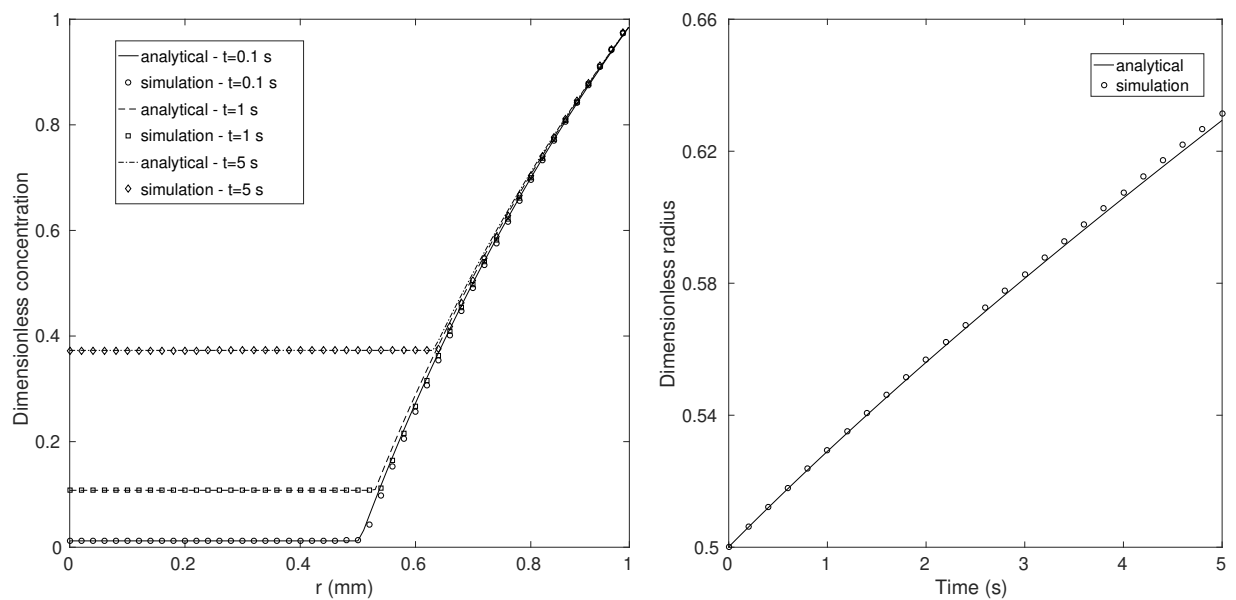

Figure 11: Comparison between numerical and analytical solution for the dimensionless concentration and dimensionless radius during growth of binary gas in infinite cylinder. 


\subsection{Mass transfer from a single-component rising gas bubble in creeping flow}

The objective of this test case is to validate the interface mass transfer in a 3D geometry with dynamic motion of the interface, on both structured and unstructured grids. For this, we consider the rising of a 3D gas bubble in the creeping flow regime $(\operatorname{Re}<<1)$. Although no analytical solution of the full species equation for this problem is available, Fleckenstein and Bothe (2015) proposed a semi-analytical method to solve the mass transfer across the interface. This uses the Hadamard-Rybczynski analytical solution (Hadamard, 1911) of flow around a spherical bubble at low Reynolds number. In spherical coordinate and in a reference frame moving with the barycentre of the bubble, the velocity field is given by

$$
\begin{gathered}
u_{\text {rad }}=-U_{b}\left(1-\frac{1.5 \mu_{d} / \mu_{c}+1}{\mu_{d} / \mu_{c}+1} \frac{1}{r / R}+\frac{\mu_{d} / \mu_{c}}{\mu_{d} / \mu_{c}+1} \frac{1}{(r / R)^{3}}\right) \cos \theta, \\
u_{\theta}=U_{b}\left(1-\frac{1.5 \mu_{d} / \mu_{c}+1}{\mu_{d} / \mu_{c}+1} \frac{1}{2 r / R}+\frac{\mu_{d} / \mu_{c}}{\mu_{d} / \mu_{c}+1} \frac{1}{2(r / R)^{3}}\right) \sin \theta,
\end{gathered}
$$

where $u_{\text {rad }}$ and $u_{\theta}$ are the radial and tangential components of the velocity, $R$ is the radius of the bubble and $U_{b}$ is the bubble rising velocity, given by

$$
U_{b}=\frac{2}{3} \frac{\rho_{d}-\rho_{c}}{\mu_{c}} g R^{2} \frac{1+\mu_{d} / \mu_{c}}{2+3 \mu_{d} \mu_{c}} .
$$

Then, assuming rotational symmetry, the stationary species equation in the continuous phase is given by

$$
u_{r} \frac{\partial c}{\partial r}+u_{\theta} \frac{1}{r} \frac{\partial c}{\partial \theta}=D\left(\frac{1}{r^{2}} \frac{\partial}{\partial r}\left(r^{2} \frac{\partial c}{\partial r}\right)+\frac{1}{\sin \theta} \frac{\partial}{\partial \theta}\left(\sin \theta \frac{\partial c}{\partial \theta}\right)\right) .
$$

Fleckenstein and Bothe (2015) numerically solves a simplified form of Eq. (70) that neglects the last term on the right-hand side by assuming assumes good mixing along the streamline, then computes the interface mass transfer to update the bubble radius $R$.

Mass transfer from a single-component gas bubble has been simulated and compared to the semi-analytical solution. The fluid properties are summarised in Table 2. A Henry's coefficient of 0.2 is chosen. A bubble with

\begin{tabular}{ccccc} 
& $\begin{array}{c}\text { Density } \\
\left(\mathrm{kg} / \mathrm{m}^{3}\right)\end{array}$ & $\begin{array}{c}\text { Dynamic viscosity } \\
(\mathrm{Pa} . \mathrm{s})\end{array}$ & $\begin{array}{c}\text { Diffusivity } \\
\left(\mathrm{m}^{2} / \mathrm{s}\right)\end{array}$ & $\begin{array}{c}\text { Interfacial tension } \\
(\mathrm{mN} / \mathrm{m})\end{array}$ \\
\hline Gas & 1.2 & $1.8 \times 10^{-5}$ & 0 & 60 \\
Liquid & 1245 & 0.46 & $1.48 \times 10^{-6}$ & \\
\hline
\end{tabular}

Table 2: Fluid properties for mass transfer from gas bubble in creeping flow

initial radius $R=2 \mathrm{~mm}$ has been simulated in a computational domain of dimension $1.2 \mathrm{~cm} \times 2.4 \mathrm{~cm} \times 1.2 \mathrm{~cm}$. Symmetry conditions are applied to the plan $\mathrm{x}=0$ and $\mathrm{z}=0$, so only a quarter of the bubble is simulated. The other boundary conditions are free-flow.

Two different grids are considered here, a structured one and an unstructured one. The structured grid is obtained by meshing the computational domain uniformly with $150 \times 300 \times 150$ cells. For the unstructured grid, a tetrahedral mesh is first obtained using the open source software gmsh (Geuzaine and Remacle, 2009) by extruding a $2 \mathrm{D}$ triangular mesh. Then, the OpenFOAM ${ }^{\circledR}$ polyDualMesh utility (OpenCFD, 2016) is used to convert to a polyhedral mesh. In order to obtain a similar number of cells for both grids, the resolution of the triangular mesh is set to $9.23 \times 10^{-5} \mathrm{~m}$ (260 points per direction). The final grids contain 6.75 million cells for the structured mesh and 6.81 million cells for the unstructured mesh.

The simulations were run with a constant time-step of $20 \mu \mathrm{s}$. Initially, the centre of the bubble is placed at ( $0 \mathrm{~mm}, 3 \mathrm{~mm}, 0 \mathrm{~mm})$. In order to compare with the semi-analytical, mass transfer from a rising bubble with no volume change and while forcing the species concentration in the bubble, and therefore the density, to remain constant is simulated until the barycentre of the bubble reaches $(0 \mathrm{~cm}, 1.2 \mathrm{~cm}, 0 \mathrm{~cm})$. The simulations are then 
restarted with local volume change taken into account. Fig. 12 shows the interface position and the dimensionless concentration $c^{*}=c /(\alpha+H(1-\alpha))$ profile at $\mathrm{t}=0.2 \mathrm{~s}$, for both structured and unstructured grids. The profile are

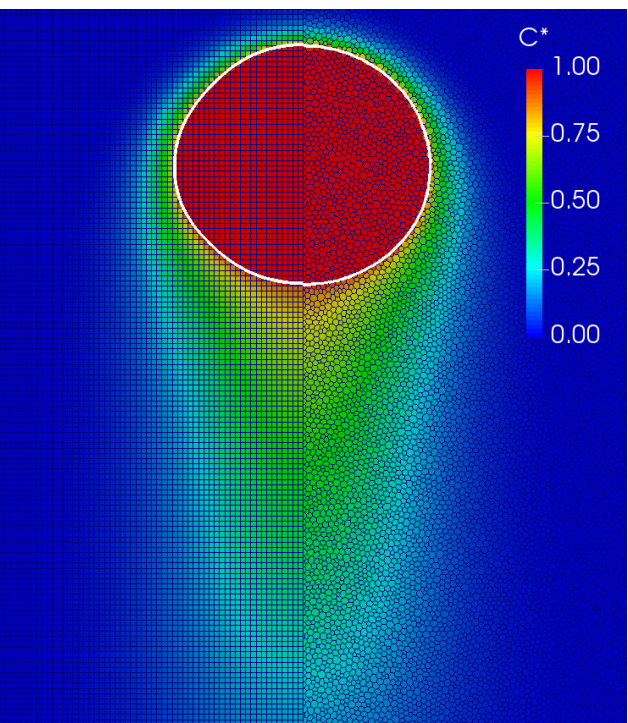

Figure 12: Interface position (white line) and dimensionless concentration profile at $\mathrm{t}=0.2 \mathrm{~s}$ for both structured and unstructured grids during rising of a gas bubble in creeping flow.

very similar. The unstructured solution is slightly more diffuse, and the bubble velocity slightly reduced, but the difference is not significant.

At $\mathrm{t}=0$, the rising velocity obtained in the simulation is equal to $3.0 \mathrm{~cm} / \mathrm{s}$, while the exact velocity obtained with the Hadamard-Rybczynski solution is approximately $3.5 \mathrm{~cm} / \mathrm{s}$. Similarly to Fleckenstein and Bothe (2015), Eq. (70) is adjusted for the semi-analytical computation to account for this discrepancy. Fig. 13 shows the evolution of the normalised mass of gas obtained with the numerical model and compared to the semi-analytical solution. After a total simulated time of $0.25 \mathrm{~s}$, the mass has decreased to $81 \%$ of its initial value in the structured grid simulation, $81.4 \%$ in the unstructured grid simulation, and to $80.6 \%$ of its initial value in the semi-analytical solution. This corresponds to a relative deviation of $0.5 \%$ for the structured grid and $1 \%$ for the unstructured grid at the end of the simulation.

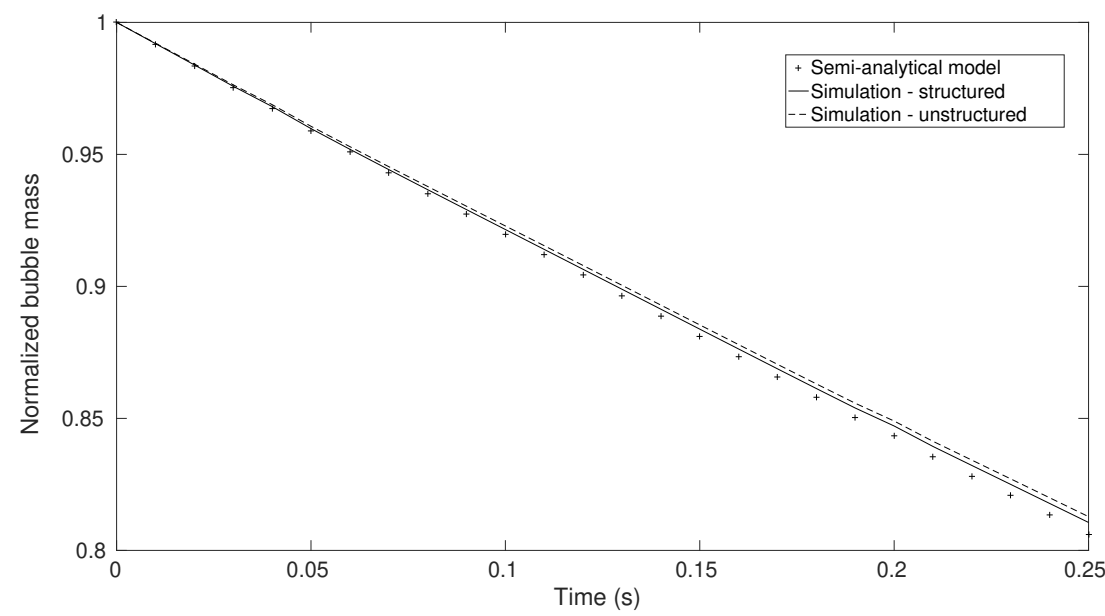

Figure 13: Comparison of semi-analytical model and numerical simulation for normalised total mass of gas during rising of a gas bubble in creeping flow. 


\section{Application}

\subsection{Competing mass transfer in infinite cylinder}

In industrial applications involving mass transfer at fluid interfaces, species being transferred from the gas phase to the liquid phase and species being transferred in the opposite direction can both happen simultaneously. This competing mass transfer directly affect the volume of each phase and must be taken into account in any numerical modelling.

Here, we consider competing mass transfer with two species A and B in an infinite cylinder of radius $\mathrm{R}=1 \mathrm{~mm}$. The solvent of the continuous phase is not soluble in the disperse phase. The fluid properties (Table 1) and the geometry (Fig. 8) are the same as in section 3.3, with the initial radius of the dispersed gas phase $R_{0}=0.5 \mathrm{~mm}$, so that the domain is filled with gas for $0 \leq r<R_{0}$ and with liquid for $R_{0}<r<R$.

Initially, the gas phase is composed at $100 \%$ of $\mathrm{B}\left(c_{A, d}=0, c_{B, d}=\rho_{d}=1 \mathrm{~kg} / \mathrm{m}^{3}\right)$, and the liquid phase is at chemical equilibrium with the gas phase $\left(c_{A, c}=0, c_{B, c}=H_{B} \rho_{d}\right)$. The diffusion coefficient are $D_{A, d}=D_{B, d}=$ $10^{-4} \mathrm{~m}^{2} / \mathrm{s}$ and $D_{A, c}=D_{B, c}=10^{-6} \mathrm{~m}^{2} / \mathrm{s}$. At the outside boundary of the system, the pressure remains constant, the concentration of $\mathrm{B}$ is equal to 0 and the concentration of $\mathrm{A}$ is equal to $H_{A} \rho_{d}$.

Like in section 3.3, the simulations are performed on a quarter-circle. The domain is represented by a $50 \times 50$ uniform Cartesian mesh. All cells containing solid are removed and replaced by rectangular and triangular cells that match the solid boundaries. The final grid contains 1875 cells. The simulation is run with a constant time-step of $1 \mu \mathrm{s}$. We consider four cases, with $H_{A}=1.0,0.5$ and $H_{B}=1.0,0.5$. The evolution of the mass fraction of A and $\mathrm{B}$ in the gas phase as well as the evolution of the gas phase radius $R_{d}$ in each case are plotted in Fig. 14.
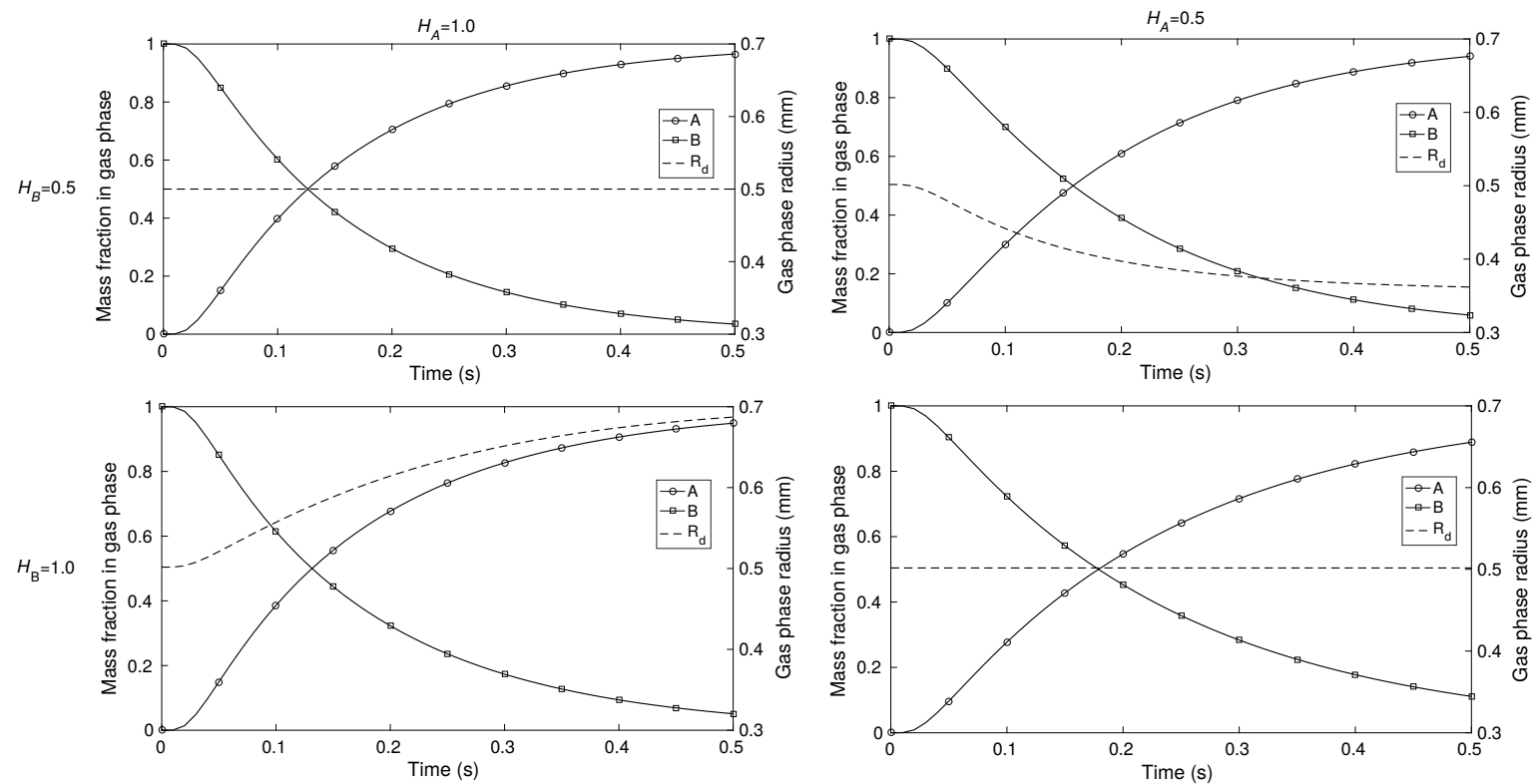

Figure 14: Evolution of mass fraction in gas phase and of gas phase radius during competing mass transfer in infinite cylinder of two component $\mathrm{A}$ and B with $H_{A}=1,0.5$ and $H_{B}=1,0.5$.

We observe that when $H_{A}=H_{B}=1.0$ and $H_{A}=H_{B}=0.5$, the radius of the gas phase remains constant. That is because when $H_{A}=H_{B}=H$, the sum of the concentration of $\mathrm{A}$ and $\mathrm{B}$ in the liquid phase is a constant equal to $H \rho_{d}$. In this case, the mass transfer fluxes at the interface are exactly opposite, and the volume of gas remains unchanged.

For the two cases when $H_{A} \neq H_{B}$, the total mass transfer is equal to zero at $\mathrm{t}=0$, since the system is initially at chemical equilibrium, but as component $\mathrm{A}$ diffuses toward the centre of the cylinder and component $\mathrm{B}$ diffuses 
away from the centre of the cylinder, the chemical balance is modified and the volume of gas change. When $H_{A}<H_{B}$, the gas phase recedes and when $H_{A}>H_{B}$, the gas phase grows. In both case, we observe that from $\mathrm{t}=0.02 \mathrm{~s}$ to $\mathrm{t}=0.1 \mathrm{~s}$, the slope is maximum and the radius changes almost linearly with time. This is because the fraction of $A$ in the gas phase is still marginal. With increasing time, the composition of the gas phase changes significantly. As the system evolves toward chemical equilibrium for component $A$, the rate of volume change decreases.

Additional multi-physics need to be included in order to model the full behaviour of the system, such as gas compressibility and pressure/temperature dependent equilibrium constant. This shall be investigated in future work.

\subsection{Mass transfer during rising of a single-component gas bubble at low Schmidt number}

In this section, the impact of the local volume change on the Sherwood number during the rising of a singlecomponent gas bubble at low Schmidt number is investigated. Two cases are considered and the fluid properties are summarised in Table 3. For test case 1, we consider a domain of size $1.2 \mathrm{~cm} \times 2.4 \mathrm{~cm} \times 1.2 \mathrm{~cm}$. A gas bubble

\begin{tabular}{ccccc} 
& $\begin{array}{c}\text { Density } \\
\left(\mathrm{kg} / \mathrm{m}^{3}\right)\end{array}$ & $\begin{array}{c}\text { Dynamic viscosity } \\
(\mathrm{Pa} . \mathrm{s})\end{array}$ & $\begin{array}{c}\text { Diffusivity } \\
\left(\mathrm{m}^{2} / \mathrm{s}\right)\end{array}$ & $\begin{array}{c}\text { Interfacial tension } \\
(\mathrm{mN} / \mathrm{m})\end{array}$ \\
\hline Gas & 1.2 & $1.8 \times 10^{-5}$ & 0 & 65 \\
Liquid 1 & 1200 & 0.024 & $2 \times 10^{-5}$ & \\
Liquid 2 & 1200 & 0.46 & $3.83 \times 10^{-4}$ & \\
\hline
\end{tabular}

Table 3: Fluid properties for mass transfer for rising of single-component gas bubble at low Schmidt number

of radius $\mathrm{R}=2 \mathrm{~mm}$ is immersed in liquid 1 (Table 3 ). The Eötvös number can be calculated using the diameter of the bubble as a reference length and we find $\mathrm{Eo}=3$. The Morton number is independent of the bubble size and $\mathrm{Mo}=10^{-5}$. For these values, Clift's diagram describing the shape regime (Clift et al., 1978) predicts an ellipsoidal shape and a Reynolds number around 30 . The bubble is initially at capillary equilibrium in the absence of gravity and interface mass transfer, with centre placed at $(0 \mathrm{~cm}, 0.3 \mathrm{~cm}, 0 \mathrm{~cm})$.

For test case 2, the domain considered and the gas bubble are five time larger $(\mathrm{R}=10 \mathrm{~mm})$, and the bubble is immersed in liquid 2 (Table 3). The Eötvös and Morton numbers are 70 and 1.3, respectively. For these values, Clift's diagram (Clift et al., 1978) predicts a dimpled ellipsoidal-cap shape and a Reynolds number around 10. Again, the bubble is initially at capillary equilibrium in the absence of gravity and interface mass transfer, with centre placed at $(0 \mathrm{~cm}, 1.5 \mathrm{~cm}, 0 \mathrm{~cm})$.

At $\mathrm{t}=0$, gravity and interface transfer are "turned on". The bubble starts rising and shrinking. Note that in both case, the diffusivity coefficient in the liquid phase corresponds to a Schmidt number $\mathrm{Sc}=1.0$.

Like in section 3.4, symmetry conditions are applied to the plan $\mathrm{x}=0$ and $\mathrm{z}=0$, so only a quarter of the bubble is simulated. The other boundary conditions are free-flow. The computational domain is resolved uniformly with $150 \times 300 \times 150$ cells. The simulation is run with a constant time-step of $20 \mu$ s for case 1 and $100 \mu$ s for case 2 . For each case, we consider two possible values of $H$ : one with $H=0.01$, so the species is only weakly dilute in the liquid phase, and one with a larger Henry's coefficient $H=0.2$. Fig. 15 show the evolution of the phase fraction during the simulations and Fig. 16 shows the species dimensionless concentration $c^{*}=c /(\alpha+H(1-\alpha))$ at the end of the simulation ( $\mathrm{t}=0.12 \mathrm{~s}$ for case $1, \mathrm{t}=0.4 \mathrm{~s}$ for case 2 ). For $H=0.01$, the bubble deforms as it rises. For case 1 , it reaches an ellipsoidal shape at $\mathrm{t}=0.05 \mathrm{~s}$, and for case 2 , it reaches a dimpled ellipsoidal-cap shape at $\mathrm{t}=0.1 \mathrm{~s}$. The impact of the bubble shape on its total surface is quantified using the shape factor Sr, defined as

$$
\mathrm{Sr}=\frac{A_{\Sigma}}{A_{\text {sphere }}} .
$$




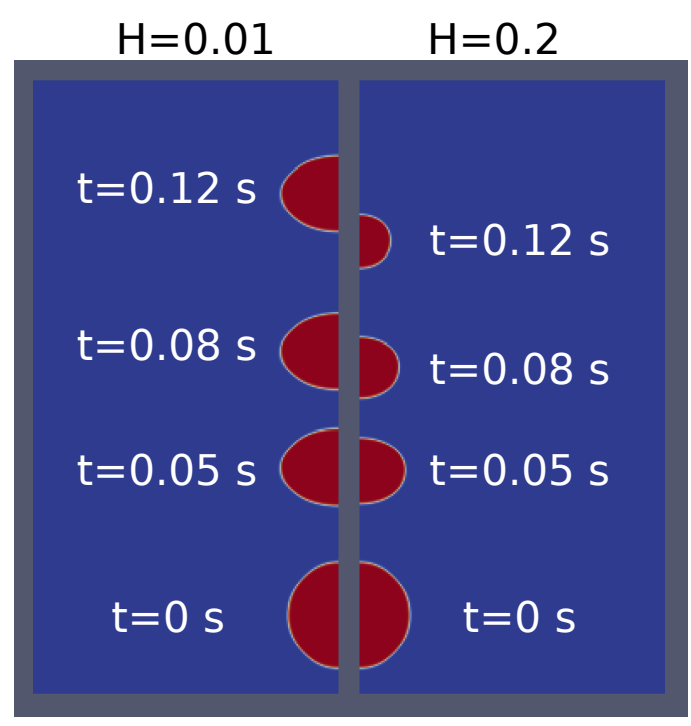

Case 1

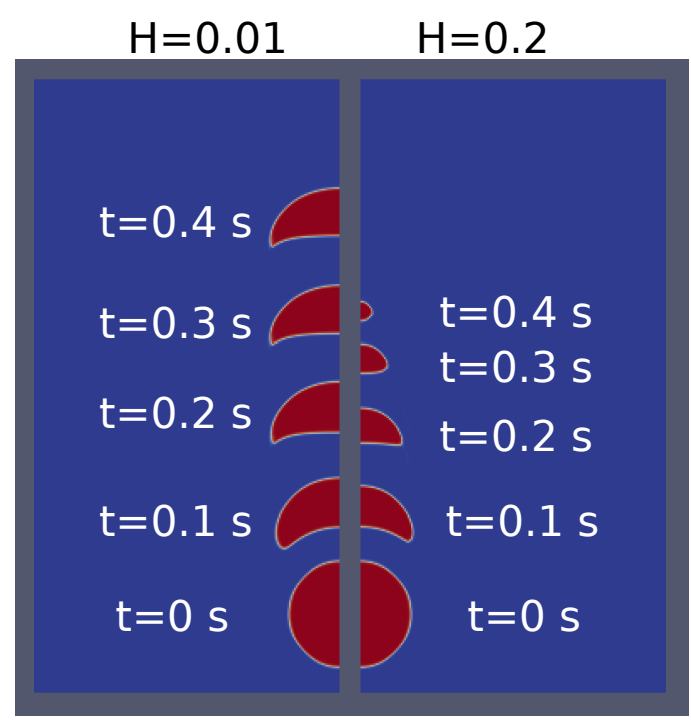

Case 2

Figure 15: Evolution of phase distribution during rising of a gas bubble for case 1 and $2, \mathrm{H}=0.01$ and $\mathrm{H}=0.2$.

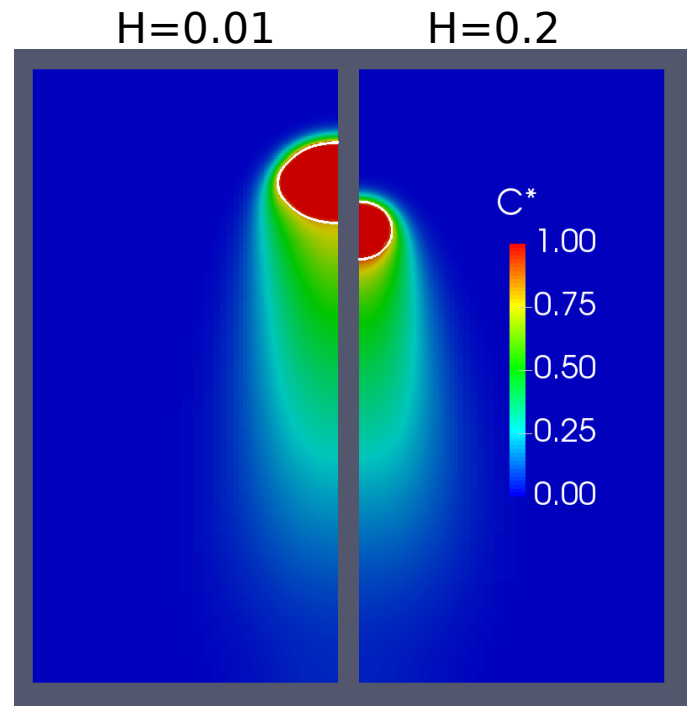

Case 1

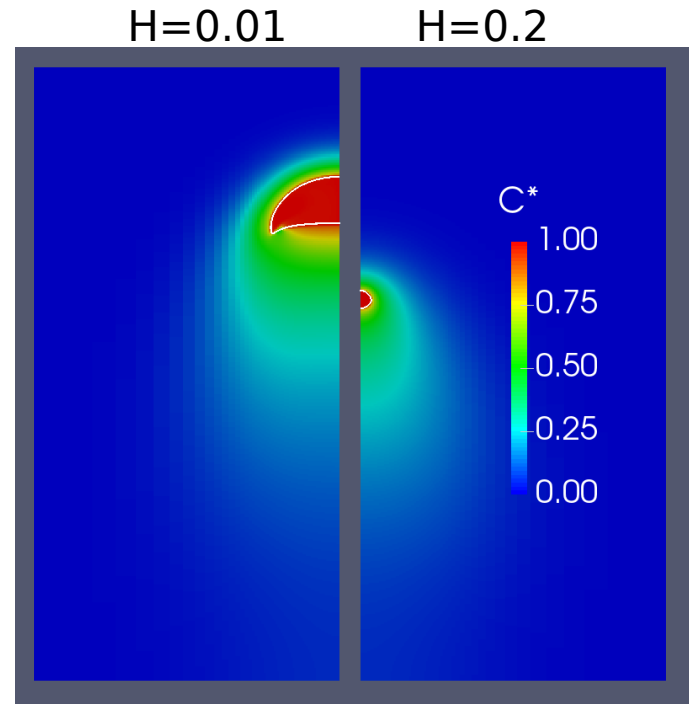

Case 2

Figure 16: Species dimensionless concentration during rising of a gas bubble for case $1(\mathrm{t}=0.12 \mathrm{~s})$ and case $2(\mathrm{t}=0.4 \mathrm{~s}), \mathrm{H}=0.01$ and $\mathrm{H}=0.2$.

The shape factor reaches a value of 1.04 for case 1 and 1.38 for case 2 . Fig. 17 shows the evolution of the Reynolds number during the simulations. The velocity of the bubble reaches a maximum of $0.155 \mathrm{~m} / \mathrm{s}$ for case 1 and 0.19 $\mathrm{m} / \mathrm{s}$ for case 2, which corresponds to Reynolds number $\mathrm{Re}=31$ and $\mathrm{Re}=9.9$. For $H=0.01$, the total loss of bubble volume at the end of the simulation is only $6 \%$ for case 1 and $8 \%$ for case 2 , so the local volume change has a limited impact on the Reynolds number. The Sherwood number is obtained by computing the mass exchange coefficient $k$ using Eq. (50). The numerical results are plotted in Fig. 18 and compared to the correlations proposed 
by Oellrich et al. (1973), with a shape factor correction (Deising et al., 2018)

$$
\begin{array}{ll}
\text { Sh.Sr }=2+0.651 \frac{\mathrm{Pe}^{1.72}}{1+\mathrm{Pe}^{1.22}} & \text { if } R e \rightarrow 0 \text { and } S c \rightarrow \infty \\
\text { Sh.Sr }=2+0.232 \frac{\mathrm{Pe}^{1.72}}{1+0.205 \mathrm{Pe}^{1.22}} & \text { if } R e \rightarrow \infty \text { and } S c \rightarrow 0
\end{array}
$$

We observe that the numerical results are closer to the case $\mathrm{Re} \rightarrow 0, \mathrm{Sc} \rightarrow \infty$, which suggests that Eq. (72) is valid for a larger parameter range. This was previously observed for mass transfer with no local volume change (Bothe and Fleckenstein, 2013; Deising et al., 2018).

For $H=0.2$, the bubble also deforms as it rises but, as gas dissolves in the liquid phase, the volume of the bubble decreases and so the Eötvös number decreases as well. For case 1, Eo is equal to 1.98 at $\mathrm{t}=0.05 \mathrm{~s}, 1.55$ at $\mathrm{t}=0.08 \mathrm{~s}$ and 1.0 at $\mathrm{t}=0.12 \mathrm{~s}$. For these values, Clift's diagram (Clift et al., 1978) predicts a transition from ellipsoidal to spherical shape, which is observed in Fig. 15. The shape factor $\mathrm{Sr}$ is equal to 1.03 at $\mathrm{t}=0.05 \mathrm{~s}, 1.02$ at $\mathrm{t}=0.08 \mathrm{~s}$ and 1.01 at $\mathrm{t}=0.12 \mathrm{~s}$. The total loss of bubble volume at the end of the simulation is $79 \%$. For case 2, Eo is equal to 45 at t=0.1 s, 27 at t=0.2 s, 13 at t=0.3 s and 3.3 at t=0.4 s. For these values, Clift's diagram (Clift et al., 1978) predicts a transition from dimpled ellipsoidal-cap to ellipsoidal shape and then from ellipsoidal to spherical shape, which is observed in Fig. 15. The shape factor $\mathrm{Sr}$ is equal $1.30 \mathrm{at} t=0.1 \mathrm{~s}, 1.22$ at $\mathrm{t}=0.2 \mathrm{~s}, 1.10$ at $\mathrm{t}=0.3 \mathrm{~s}$ and 1.08 at $\mathrm{t}=0.4 \mathrm{~s}$. The total loss of bubble volume at the end of the simulation is $99 \%$.

Moreover, the bubble velocity decreases as it dissolves. Fig. 17 shows the evolution of the Reynolds number during the simulations. We observe that the bubble does not reach the same maximum Reynolds $(\operatorname{Re}=31$ for case 1 and $\operatorname{Re}=10$ for case 2) as for $H=0.01$. Instead, the Reynolds reaches a maximum $\operatorname{Re}=24$ at $\mathrm{t}=0.04 \mathrm{~s}$ for case 1 and $\mathrm{Re}=8.9$ at $\mathrm{t}=0.08 \mathrm{~s}$ for case 2 , and then decreases almost linearly. Fig. 18 shows a comparison between the Sherwood number numerically obtained and the ones obtained from Oellrich correlations (Eqs. (72) and (73)).
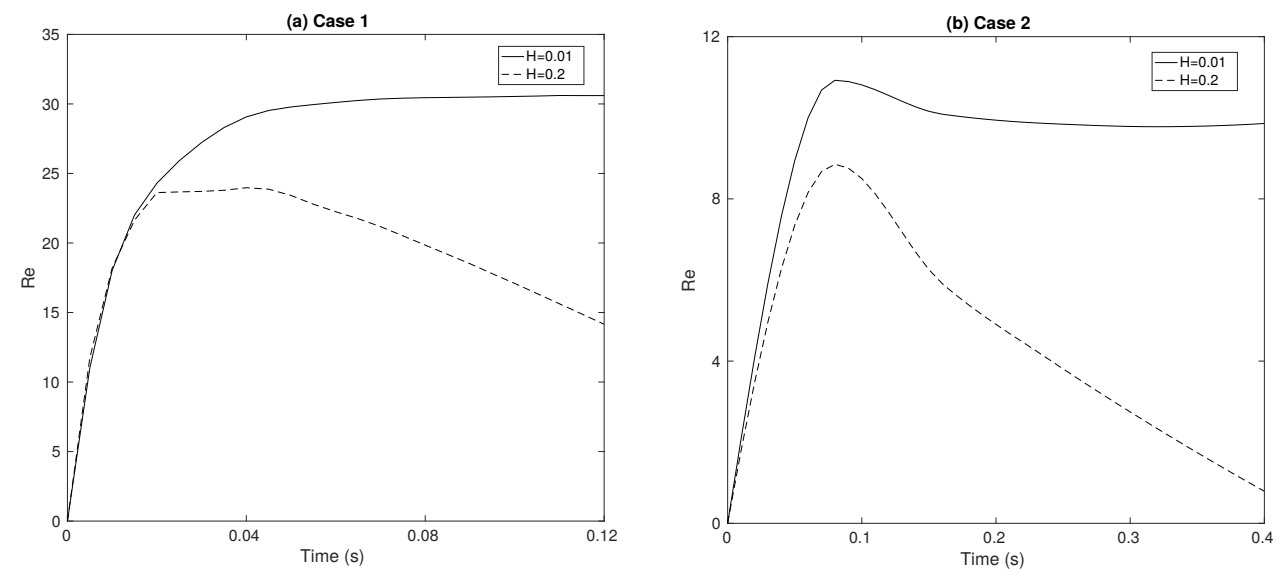

Figure 17: Evolution of Reynolds number during rising of a gas bubble for case 1 and 2, $\mathrm{H}=0.01$ and $\mathrm{H}=0.2$.

Again, we observe that the numerical results are closer to the case $\mathrm{Re} \rightarrow 0, \mathrm{Sc} \rightarrow \infty$. Moreover, the trend of the Sherwood number which decreases as the bubble volume decreases is accurately represented. This suggests that the impact of local volume change on Sherwood number might be accurately described by using the timedependent Reynolds number in the same integral correlations.

\section{Conclusion}

In this paper, a novel unified single-field formulation for Volume-Of-Fluid simulation of interfacial mass transfer with local volume changes has been presented. The main focus of this work is a rigorous derivation of the 

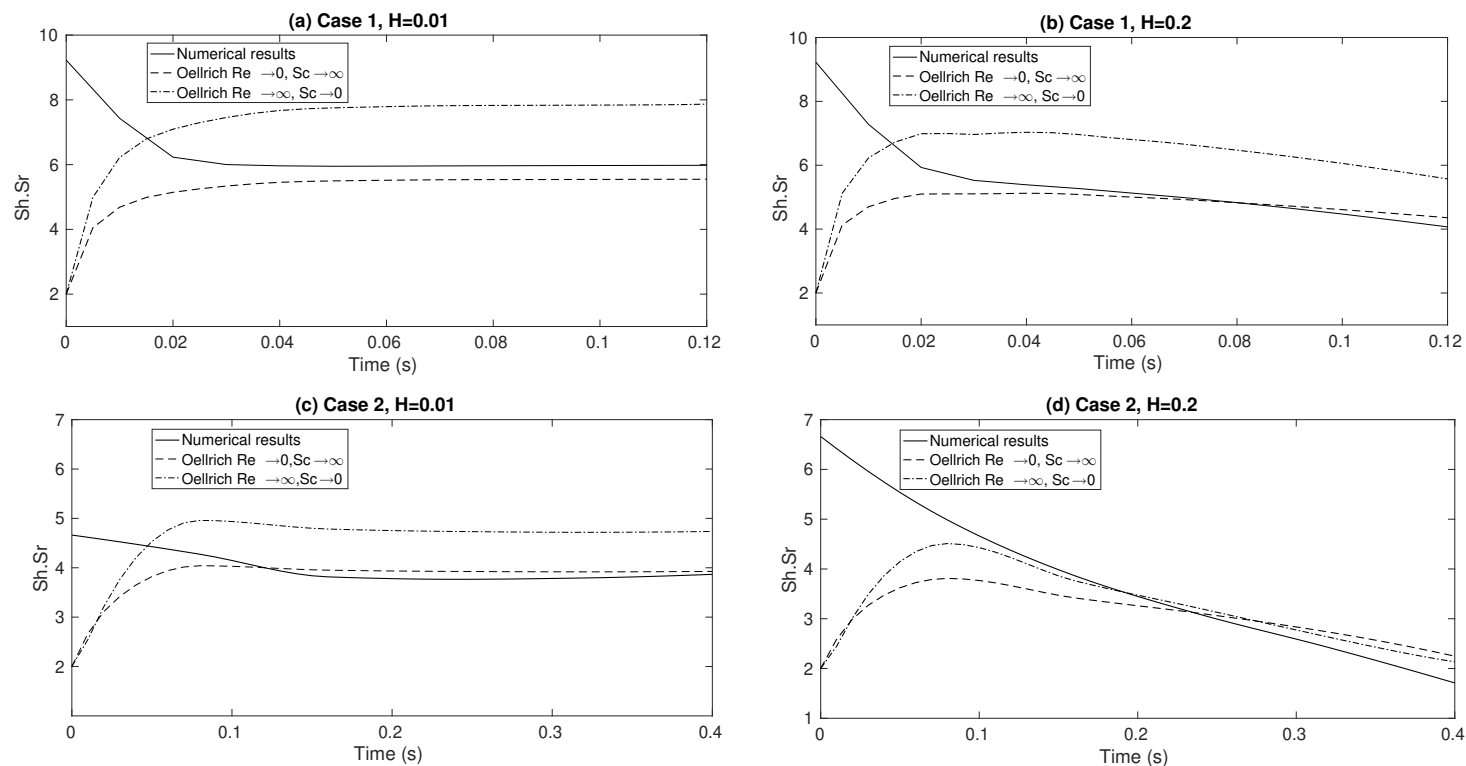

Figure 18: Evolution of Sherwood number and comparison with Oellrich correlation during rising of a gas bubble for case 1 and 2 , $\mathrm{H}=0.01$ and $\mathrm{H}=0.2$.

single-field local mass transfer between phases at the interface, which is re-injected in the phase conservation equation. To compute this mass transfer and the local volume change, both convective and diffusive contribution are considered. The single-field formulation couples the mass and momentum transfer via a source term in the pressure equation.

The numerical method is validated by comparison with four test cases. For the first test case, artificial mass transfer is evaluated for a case with infinite Péclet number, and we observed consistency between advection of phase fraction and advection of species concentration in the case of an algebraic VOF method with compression of the interface using the normal C-CST method. For the second and third test cases, analytical solutions of the dissolution of a single-component gas phase into an immiscible liquid solvent in a semi-infinite tube and of the growth of a binary gas phase in an infinite cylinder are considered. For the last test case, we use the semianalytical solution described by Fleckenstein and Bothe (2015) for the mass transfer across the interface of a rising gas bubble in the creeping flow regime. A very good agreement of the numerical results with the reference solution is obtained in each case. The main weakness of the approach is the first-order accurate discretization of the interface transfer $\dot{m}$. Additional research is needed to develop a bounded second-order discretization.

The validated approach is first applied to investigate competing mass transfer between two species A and B in an infinite cylinder. The simulation was able to reproduce the direction of the gas/liquid interface motion for various cases where $H_{A}=H_{B}, H_{A}<H_{B}$ and $H_{B}<H_{A}$, as well as the impact of total mass fraction in the bubble. In future work, additional multi-physics such as gas compressibility and pressure/temperature dependent equilibrium constant will be included.

Finally, the method is applied to investigate mass transfer during the rising of a single-component gas bubble at low Schmidt number. Our results suggests that the impact of local volume change on the Sherwood number might be accurately described by using the time-dependent Reynolds number in the same integral correlations.

By comparison with the two-field method presented by Fleckenstein and Bothe (2015), the advantage of the single-field formulation is that it is fully consistent in principles with any algebraic VOF method, as long as the same discretization schemes are applied for all advected quantities. We showed that within a VOF method with compression of the interface, it can be applied on structured and unstructured grids, with comparable results. In future work, our numerical model will be used to investigate dissolution during geological trapping of $\mathrm{CO}_{2}$ in 
580 aquifers, for which unstructured grids are essential to capture the complex geometry of the porous media and

${ }_{581}$ accurately estimate key parameters such as pore surface area (Graveleau et al., 2017; Soulaine et al., 2017).

582

583

584

58

586 Variables

${ }_{587} A \quad$ Area $\left(\mathrm{m}^{2}\right)$

${ }_{588} \quad c \quad$ concentration $\left(\mathrm{kg} / \mathrm{m}^{3}\right)$

${ }_{589} \quad D \quad$ molecular diffusivity $\left(\mathrm{m}^{2} / \mathrm{s}\right)$

${ }_{590} \quad F \quad$ advective flux $\left(\mathrm{kg} / \mathrm{m}^{2} / \mathrm{s}\right)$

${ }_{591} \quad f \quad$ interior force $\left(\mathrm{N} / \mathrm{m}^{3}\right)$

$592 \quad g \quad$ gravity accelaration $\left(\mathrm{m}^{2} / \mathrm{s}\right)$

${ }_{593} \quad H \quad$ Henry constant

${ }_{594} \quad J \quad$ molecular diffusion flux $\left(\mathrm{kg} / \mathrm{m}^{2} / \mathrm{s}\right)$

${ }_{595} k \quad$ mass exchange coefficient $(\mathrm{m} / \mathrm{s})$

$596 \quad l \quad$ length $(\mathrm{m})$

$597 \quad$ normal vector

${ }_{598} p \quad$ pressure $(\mathrm{Pa})$

$599 \quad r \quad$ radius $(\mathrm{m})$

$600 S \quad$ Stress tensor $\left(\mathrm{kg} / \mathrm{m} / \mathrm{s}^{2}\right)$

$601 \quad t \quad$ time (s)

$602 \quad$ velocity $(\mathrm{m} / \mathrm{s})$

$603 \quad V \quad$ Volume $\left(\mathrm{m}^{3}\right)$

$604 w \quad$ interface velocity $(\mathrm{m} / \mathrm{s})$

${ }_{605} \alpha \quad$ phase volume fraction

$606 \quad \kappa \quad$ interface curvature $\left(\mathrm{m}^{-1}\right)$

$607 \quad \mu \quad$ viscosity (Pa.s)

$608 \Phi \quad$ flux $\left(\mathrm{kg} / \mathrm{m}^{2} 3 / \mathrm{s}\right)$

$609 \rho \quad$ mass density $\left(\mathrm{kg} / \mathrm{m}^{3}\right)$ 


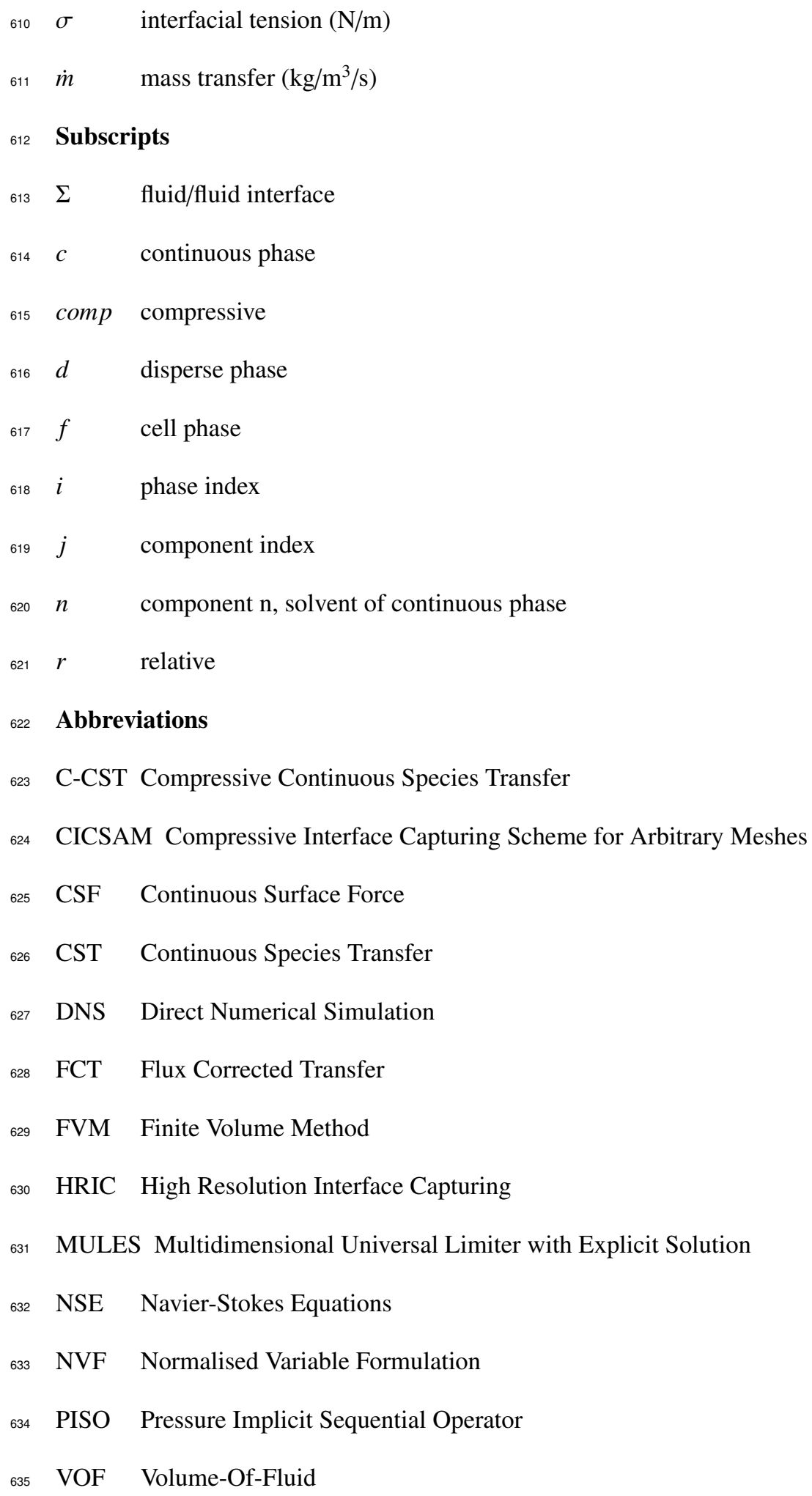




\section{References}

Albadawi, A., Donoghue, D. B., Robinson, A. J., Murray, D. B., Delaure, Y. M. C., 2013. Influence of surface tension implementation in Volume Of Fluid and coupled Volume Of Fluid with Level Set method for bubble growth and detachment. Int. J. Multiphase Flow 53, 11-28.

Bothe, D., Fleckenstein, S., 2013. A volume-of-fluid-based method for mass transfer processes at fluid particles. Chem. Eng. Sci. 101, 283302.

Brackbill, J. U., Kothe, D. B., Zemach, C., 1992. A continuum method for modeling surface tension. J. Comput. Phys. 100 (2), $335-354$.

Chai, M., Luo, K., Shao, C., Fan, J., 2017. An efficient level set remedy approach for simulaion of two-phase flow based on sigmoid function. Chem. Eng. Sci. 172, 335-352.

Clift, R., Grace, J. R., Weber, M. E., 1978. Bubbles, drops, and particles. New York; London: Academic Press.

Coutelieris, F., Kainourgiakis, M., Stubos, A., Kikkinides, E., Yortsos, Y., 2006. Multiphase mass transport with partitioning and inter-phase transport in porous media. Chem. Eng. Sci. 61 (14), 4650-4661.

Danckwerts, P. V., 1970. Gas-liquid reaction. McGraw-Hill, New-York, New-York.

Deising, D., Bothe, D., Marschall, H., 2018. Direct numerical simulation of mass transfer in bubbly flows. Computer and Fluids $172,524-537$.

Deising, D., Marschall, H., Bothe, D., 2016. A unified single-field model framework for Volume-Of-Fluid simulations of interfacial species transfer applied to bubbly flow. Chem. Eng. Sci. 139, 173-195.

Fleckenstein, S., Bothe, D., 2015. A volume-of-fluid-based numerical method for multi-component mass transfer with local volume changes. J. Comput. Phys. 301, 35-58.

Francois, M. M., Cummins, S. J., Dendy, E. D., Kothe, D. B., Sicilan, J. M., Williams, M. W., 2006. A balanced-force algorithm for continuous and sharp interfacial surface tension models within a volume tracking framework. J. Comput. Phys. 213 (1), $141-173$.

Gerlach, D., Tomar, G., Biswas, G., Durst, F., 2006. Comparison of volume-of-fluid method for surface tension-dominant two-phase flows. Int. J. Heat Mass Transfer 49, 740-754.

Geuzaine, C., Remacle, J.-F., 2009. Gmsh: A 3-d finite element mesh generator with built-in pre-and post-processing facilities. International Journal for Numerical Methods in Engineering 79 (11), 1309-1331.

Gibou, F., Fedkiw, R., Osher, S., 2018. A review of level-set method and some recent applications. J. Comput. Phys. 353, 82-109.

Graveleau, M., Soulaine, C., Tchelepi, H., 2017. Pore scale simulation of interface multicomponent mass transfer for subsurface flow. Transport in porous media $120(2), 287-308$.

Hadamard, J., 1911. Mouvement permanent lent d'une sphère liquide et visqueuse dans un liquide visqueux. C.R. Acad. Sci. Paris 152, $1735-1738$.

Haroun, Y., Legendre, D., Raynal, L., 2010. Volume of fluid method for interfacial reactive mass transfer: Application to stable liquid film. Chem. Eng. Sci. 65 (10), 2896-2909.

Hirt, C. W., Nichols, B. D., 1981. Volume-Of-Fluid (VOF) method for the dynamic of free boundaries. J. Comput. Phys. 39 (1), $201-225$.

Issa, R., Ahmadi-Befrui, B., Beshay, K., Gosman, A., 1985. Solution of the implicitly discretised reacting flow equations by operator-splitting. J. Comput. Phys. 93 (2), 388-410.

Leonard, B. P., 1988. Universal limiter for transient interpolation modeling of the advective transport equations: the ultimate conservative differencing scheme. NASA Technical Memorandum 100916.

Luo, K., Shao, C., Chai, M., Fan, J., 2019. Level set method for atomization and evaporation simulations. Progress in Energy and Combustion Science 50, 49-75.

Maes, J., Geiger, S., 2018. Direct pore scale reactive transport modelling of dynamic wettability changes induced by surface complexation in carbonate rocks. Adv. iWater Resour. 111, 6-19.

Maes, J., Soulaine, C., 2018. A new compressive scheme to simulate species transfer across fluid interfaces using the volume-of-fluid method. Chem. Eng. Sci. 190 (23), 405-418.

Maric, T., Marschall, H., Bothe, D., 2013. voFoam - A geometrical Volume-Of-Fluid Algorithm on Arbitrary Unstructured Meshes with Local Dynamic Adaptive Mesh Refinement Using OpenFOAM. ArXiv e-prints.

Marschall, H., Hinterberger, K., Schüler, C., Habla, F., Hinrichsen, O., 2012. Numerical simulation of species transfer across fluid interfaces in free-surface flows using OpenFOAM. Chemical Engineering Science 78, 111-127.

Muzaferija, S., Peric, M., Sames, P., Schellin, T., 1999. A two-fluid navier-stokes solver to simulate water entry. Twenty-Second Symposium on Naval Hydrodynamics, 638-664.

Oellrich, H., Schmidt-Traub, H., Brauer, H., 1973. Theoretische berechnung des stofftransports in der umgebung einer einzelblase. Chem. Eng. Sci. 28, 711-721.

OpenCFD, 2016. OpenFOAM, the open source cfd toolbox, User Guide. OpenCFD Ltd.

Owkes, M., Desjardins, O., 2014. A computational framework for conservative, three-dimensional, unsplit, geometric transport with application to the volume-of-fluid (vof) method. J. Comput. Phys. 270, 587-612.

Patankar, S. V., 1980. Numerical Heat and Mass Transfer. Hemisphere Publ. Corp., Washington.

Popinet, S., 2009. An accurate adaptive solver for surface-tension-driven interfacial flows. J. Comput. Phys. 228, 5838-5866.

Popinet, S., 2018. Numerical models of surface tension. Annual review of fluid mechanics 50, 49-76. 
Quintard, M., Whitaker, S., 1994. Convection, dispersion, and interfacial transport of contaminants: Homogeneous porous media. Adv. Water Resour. 17 (4), 116-126.

Raeini, A., Blunt, M. J., Bijeljic, B., 2012. Modelling two-phase flow in porous media at the pore-scale using the volume-of-fluid method. J. Comput. Phys. 231 (17), 5653-5668.

Rusche, H., 2002. Computational fluid dynamics of dispersed two-phase flows at high phase fraction. Ph.D. thesis, Imperial College London. Scardovelli, R., Zaleski, S., 1999. Direct numerical simulation of free-surface and interfacial flow. Annu. Rev. Fluid Mech. 31 , 567-603.

Soh, G. Y., Yeoh, G. H., Timchenko, V., 2017a. A cfd model for the coupling of multiphase, multicomponent and mass transfer physics for micro-scale simulation. International Journal of Heat and Mass Transfer 113, 922-934.

Soh, G. Y., Yeoh, G. H., Timchenko, V., 2017b. Numerical investigation of formation and dissolution of $\mathrm{co}_{2}$ bubbles within silicone oil in cross-junction microchannel. Microfluid Nanofluid 21 (175).

Soulaine, C., Creux, P., Tchelepi, H. A., 2019. Micro-continuum framework for pore-scale multiphase fluid transport in shale formations. Transp. Porous Med. 127 (1), 85-112.

Soulaine, C., Debenest, G., Quintard, M., 2011. Upscaling multi-component two-phase flow in porous media with partitioning coefficient. Chem. Eng. Sci. 66 (23), 6180-6192.

Soulaine, C., Roman, S., Kovscek, A., Tchelepi, H. A., 2017. Mineral dissolution and wormholing from a pore scale perspective. J. Fluid Mech. 827, 457-483.

Soulaine, C., Roman, S., Kovscek, A., Tchelepi, H. A., 2018. Pore-scale modelling of multiphase reactive flow. application to mineral dissolution with production of co2. J. Fluid Mech. 855, 616-645.

Sussman, M., Smereka, P., Osher, S., 1994. A level-set approach for computing solutions to incompressible tow-phase flow. J. Comput. Phys. $114,146-159$.

Takemura, F., Yabe, A., 1998. Gas dissolution process of spherical rising gas bubbles. Chem. Eng. Sci. 53 (15), $2691-2699$.

Taylor, R., Krishna, R., 1993. Multicomponent mass transfer. Vol. 2. John Wiley \& Sons.

Ubbink, O., Issa, R. I., 1999. A method for capturing sharp fluid interfaces on arbitrary meshes. J. Comput. Phys. 153, 26-50.

van Leer, B., 1974. Towards the ultimate conservative difference scheme. II. Monotonicity and conservation combined in a second-order scheme. J. Comput. Phys. 14 (4), 361-370.

Weller, H. G., 2006. A new approach to vof-based interface capturing method for incompressible and compressible flow. Technical ReportOpenCFD Limited.

Weymouth, G. D., Yue, D. K. P., 2010. Conservative volume-of-fluid method for free-surface simulations on cartesian grids. J. Comput. Phys. 229 (8), 2853-2865.

Whitaker, S., 1999. The method of volume averaging, theory and applications of transport in porous media. Dorderecht: Kluwer Academic.

Yang, L., Nieves-Remacha, M. J., Jensen, K. F., 2017. Simulations and analysis of multiphase transport and reaction in segmented flow microreactors. Chem. Eng. Sci. 169, 106-116.

Zalesak, S. T., 1979. Fully multidimensional flux-corrected transport algorithm for fluids. J. Comput. Phys. 31, 335-362. 